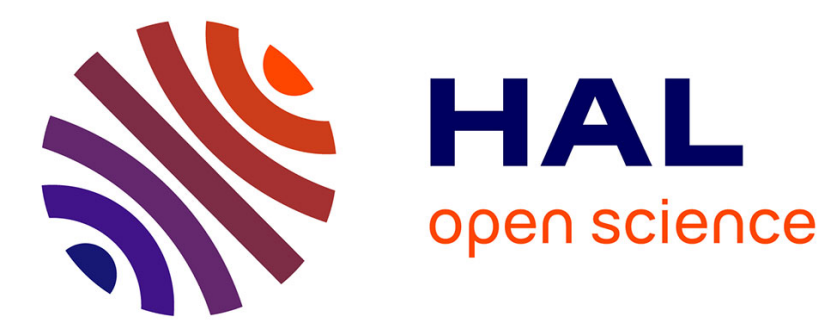

\title{
Compactness of discrete approximate solutions to parabolic PDEs - Application to a turbulence model
}

Thierry Gallouët, J.-C Latché

\section{To cite this version:}

Thierry Gallouët, J.-C Latché. Compactness of discrete approximate solutions to parabolic PDEs Application to a turbulence model. Communications on Pure and Applied Analysis, 2012, 11 (6), pp.21. 10.3934/cpaa.2012.11.2371 . hal-01283559

\section{HAL Id: hal-01283559 \\ https://hal.science/hal-01283559}

Submitted on 7 Mar 2016

HAL is a multi-disciplinary open access archive for the deposit and dissemination of scientific research documents, whether they are published or not. The documents may come from teaching and research institutions in France or abroad, or from public or private research centers.
L'archive ouverte pluridisciplinaire HAL, est destinée au dépôt et à la diffusion de documents scientifiques de niveau recherche, publiés ou non, émanant des établissements d'enseignement et de recherche français ou étrangers, des laboratoires publics ou privés. 


\title{
COMPACTNESS OF DISCRETE APPROXIMATE SOLUTIONS TO PARABOLIC PDES - APPLICATION TO A TURBULENCE MODEL
}

\author{
T. GALlouËT \\ Université de Provence \\ CMI \\ Marseille, France \\ J.-C. LATChÉ \\ Institut de Radioprotection et de Sûreté Nucléaire \\ BP 3, 13115 Saint-Paul-lez-Durance Cedex, France
}

(Communicated by the associate editor name)

\begin{abstract}
In this paper, we prove an adaptation of the classical compactness Aubin-Simon lemma to sequences of functions obtained through a sequence of discretizations of a parabolic problem. The main difficulty tackled here is to generalize the classical proof to handle the dependency of the norms controlling each function $u^{(n)}$ of the sequence with respect to $n$. This compactness result is then used to prove the convergence of a numerical scheme combining finite volumes and finite elements for the solution of a reduced turbulence problem.
\end{abstract}

1. Introduction. Let us suppose given a sequence of approximations of a parabolic problem $(\mathcal{P})$, which, for instance, may be though of as discretizations of $(\mathcal{P})$ by a numerical scheme, or resulting from the combination of a Faedo-Galerkin technique with a time discretization. In both cases, we are in presence of a family of finitedimensional systems, and their solution, let us say $\left(u^{(n)}\right)_{n \in \mathbb{N}}$, may be considered as a sequence of functions of time, taking their values in a finite dimensional subspace, let us say $B^{(n)}$ of a Banach space (usually a Lebesgue $\mathrm{L}^{q}$ space, $q \geq 1$ ). To show the convergence of such a process, a common path is to follow the following steps:

1. first, for each approximate problem, prove the existence of a solution, and derive estimates satisfied by any solution,

2. then use compactness arguments to show (possibly up to the extraction of a subsequence) the existence of a limit,

3. and, finally, prove that this limit satisfies the initial problem $(\mathcal{P})$.

Let us now focus on item 2 , which is the issue addressed in this paper. The problem here is to prove a compactness result for a sequence of functions of time taking their value in a sequence of discrete spaces and controlled (themselves and their discrete time-derivative) in discrete norms; in the general case, both $B^{(n)}$ and the space part of the norms depend on $n$. The compactness result given in this paper relies on this particular structure, and consists in a generalization of the classical Aubin-Simon lemma to this specific case.

2000 Mathematics Subject Classification. Primary: 58F15, 58F17; Secondary: 53C35.

Key words and phrases. Partial differential equations, discretization, convergence. 
This paper is organized as follows. First, we recall some classical compactness lemmas (Section 2). Then, in the next section (Section 3), we state and prove the main result of this paper. Finally, the way to use this result is illustrated (Section 4), by proving the convergence of a scheme combining finite element and finite volume techniques to solve a reduced turbulence model.

2. A classical compactness lemma. A classical way to obtain compactness of a sequence of approximate solutions of an evolution problem is given by the wellknown Aubin-Simon Theorem [14], which is a consequence of a compactness criterium due to Kolmogorov. We begin by recalling these results.

Theorem 2.1. Let $B$ be a Banach space, $1 \leq q<+\infty$ and $\mathcal{F} \subset \mathrm{L}^{q}((0, T), B)$. If $\mathcal{F}$ satisfies the following conditions:

1. For all $f \in \mathcal{F}$, there exists $P f \in \mathrm{L}^{q}(\mathbb{R}, B)$ such that $P f=f$ a.e. in $(0, T)$ and $\|P f\|_{L^{q}(\mathbb{R}, B)} \leq C$, where $C$ depends only on $\mathcal{F}$.

2. For all $\varphi \in \mathrm{C}_{c}^{\infty}(\mathbb{R}, \mathbb{R})$, the family $\left\{\int_{\mathbb{R}}(P f) \varphi \mathrm{d} t, f \in \mathcal{F}\right\}$ is relatively compact in $B$.

3. $\|P f(\cdot+h)-P f\|_{\mathrm{L}^{q}(\mathbb{R}, B)} \rightarrow 0$, as $h \rightarrow 0$, uniformly with respect to $f \in \mathcal{F}$, then the subset $\mathcal{F}$ is relatively compact in $\mathrm{L}^{q}((0, T), B)$.

Remark 1. Note that the conditions given in Theorem 2.1 are in fact necessary: if the subset $\mathcal{F}$ is relatively compact in $\mathrm{L}^{q}((0, T), B)$, then items $1-3$ hold. In addition, it is even possible to define $P f$ by extending $f$ by zero outside $(0, T)$. The proof of this implication is quite simple and omitted here because the useful part (at least for the present work) of this equivalence is the one stated in Theorem 2.1.

Proof. Let $\left(\rho^{(n)}\right)_{n \in \mathbb{N}^{*}}$ be a sequence of mollifiers, that is:

$$
\begin{aligned}
\rho \in \mathrm{C}_{c}^{\infty}(\mathbb{R}, \mathbb{R}), \int_{\mathbb{R}} \rho \mathrm{d} \boldsymbol{x}=1, \rho \geq 0 \text { in } \mathbb{R}, \rho=0 \text { for } x \notin(-1,1) \\
\text { and, for } n \in \mathbb{N}^{\star}, \boldsymbol{x} \in \mathbb{R}, \rho^{(n)}(\boldsymbol{x})=n \rho(n \boldsymbol{x}) .
\end{aligned}
$$

We set $K=[0, T]$ and, for $n \in \mathbb{N}^{\star}, \mathcal{F}^{(n)}=\left\{\left(P f \star \rho^{(n)}\right)_{\left.\right|_{K}}, f \in \mathcal{F}\right\}$. The proof is divided in two steps. In Step 1 we prove, using the Ascoli theorem and Assumption 2 of Theorem 2.1, that, for $n \in \mathbb{N}^{\star}$, the set $\mathcal{F}^{(n)}$ is relatively compact in $\mathrm{C}(K, B)$ endowed with its usual topology. Then, we deduce that $\mathcal{F}^{(n)}$ is also relatively compact in $\mathrm{L}^{q}((0, T), B)$. In Step 2, we show that the first and third hypotheses of Theorem 2.1 give that $P f \star \rho^{(n)} \rightarrow P f$ in $\mathrm{L}^{q}(\mathbb{R}, B)$, as $n \rightarrow+\infty$, uniformly with respect to $f \in \mathcal{F}$. This allows to conclude that the family $\mathcal{F}$ is relatively compact in $\mathrm{L}^{q}((0, T), B)$.

Step 1. Let $n \in \mathbb{N}^{\star}$. In order to prove that $\mathcal{F}^{(n)}$ is relatively compact in $\mathrm{C}(K, B)$, we have only to prove, thanks to the Ascoli theorem, the following properties:

1. For all $t \in K$, the set $\left\{P f \star \rho^{(n)}(t), f \in \mathcal{F}\right\}$ is relatively compact in $B$,

2. the sequence $\left\{P f \star \rho^{(n)}, f \in \mathcal{F}\right\}$ is equicontinuous from $K$ to $B$ (that is to say that the continuity is uniform with respect to $f \in \mathcal{F})$.

We first prove Property 1. Let $t \in K$. We set, for $s \in \mathbb{R}, \varphi(s)=\rho^{(n)}(t-s)$. We have $\varphi \in \mathrm{C}_{c}^{\infty}(\mathbb{R}, \mathbb{R})$ and for all $f \in \mathcal{F}$,

$$
P f \star \rho^{(n)}(t)=\int_{\mathbb{R}} \operatorname{Pf}(s) \rho^{(n)}(t-s) \mathrm{d} s=\int_{\mathbb{R}} \operatorname{Pf}(s) \varphi(s) \mathrm{d} s .
$$


Then, the second hypothesis of Theorem 2.1 gives Property 1, i.e. $\left\{P f \star \rho^{(n)}(t), f \in\right.$ $\mathcal{F}\}$ is relatively compact in $B$.

We now turn to Property 2. Let $t_{1}, t_{2} \in K$. One has, with $r=q /(q-1)$ and using Hölder's Inequality,

$$
\begin{array}{r}
\left\|P f \star \rho^{(n)}\left(t_{2}\right)-P f \star \rho^{(n)}\left(t_{1}\right)\right\|_{B} \leq \int_{\mathbb{R}}\|P f(s)\|_{B}\left|\rho^{(n)}\left(t_{2}-s\right)-\rho^{(n)}\left(t_{1}-s\right)\right| \mathrm{d} s \\
\leq\|P f\|_{\mathrm{L}^{q}(\mathbb{R}, B)}\left\|\rho^{(n)}\left(t_{2}-\cdot\right)-\rho^{(n)}\left(t_{1}-\cdot\right)\right\|_{\mathrm{L}^{r}(\mathbb{R})} .
\end{array}
$$

Since $\rho^{(n)}$ is uniformly continuous with compact support, we easily deduce from this inequality (and with Hypothesis 1 of Theorem 2.1) that the family $\mathcal{F}^{(n)}$ is uniformly equicontinuous from $K$ to $B$. This gives Property 2 and proves that $\mathcal{F}^{(n)}$ is relatively compact in $\mathrm{C}(K, B)$. This relative compactness is equivalent to say that for all $\epsilon>0$, there exists a finite number of balls of radius $\epsilon$ (for the natural norm of $\mathrm{C}(K, B))$ covering the whole set $\mathcal{F}^{(n)}$. Then, since $\|\cdot\|_{\mathrm{L}^{q}((0, T), B)} \leq T^{1 / q}\|\cdot\|_{\mathrm{C}(K, B)}$, we also obtain the relative compactness of $\mathcal{F}^{(n)}$ in $\mathrm{L}^{q}((0, T), B)$.

Step 2. For $t \in \mathbb{R}$, we have, using $\int_{\mathbb{R}} \rho^{(n)}(s) \mathrm{d} s=1$ and with $\bar{s}=n s$,

$$
\begin{array}{r}
P f \star \rho^{(n)}(t)-P f(t)=\int_{\mathbb{R}}(P f(t-s)-P f(t)) \rho^{(n)}(s) \mathrm{d} s= \\
\int_{-1}^{1}\left(P f\left(t-\frac{\bar{s}}{n}\right)-P f(t)\right) \rho(\bar{s}) \mathrm{d} \bar{s} .
\end{array}
$$

Then, with Hölder's Inequality and $r=q /(q-1)$,

$$
\left\|P f \star \rho^{(n)}(t)-P f(t)\right\|_{B}^{q} \leq\left(\int_{-1}^{1}\left\|P f\left(t-\frac{s}{n}\right)-P f(t)\right\|_{B}^{q} \mathrm{~d} s\right)\|\rho\|_{\mathrm{L}^{r}(\mathbb{R})}^{q} .
$$

Integrating with respect to $t \in \mathbb{R}$ and using the Fubini-Tonelli theorem leads to:

$$
\begin{aligned}
& \left\|P f \star \rho^{(n)}-P f\right\|_{L^{q}(\mathbb{R}, B)}^{q} \leq \\
& \quad 2 \sup \left\{\|P f(\cdot+h)-P f\|_{L^{q}(\mathbb{R}, B)}^{q},|h| \leq 1 / n\right\}\|\rho\|_{L^{r}(\mathbb{R})}^{q} .
\end{aligned}
$$

Finally, the third hypothesis of Theorem 2.1 gives the fact that $\| P f \star \rho^{(n)}-$ $P f \|_{\mathrm{L}^{q}(\mathbb{R}, B)} \rightarrow 0$, as $n \rightarrow+\infty$, uniformly with respect to $f \in \mathcal{F}$. As a consequence, the relative compactness of $\mathcal{F}^{(n)}$ in $\mathrm{L}^{q}((0, T), B)$ for all $n \in \mathbb{N}$ (proven in Step 1) gives the relative compactness of $\mathcal{F}$ in $\mathrm{L}^{q}((0, T), B)$. This concludes the proof of Theorem 2.1.

Theorem 2.2. Let $1 \leq q<+\infty$ and $X, B, Y$ be three Banach spaces such that:

$-X \subset B$ with compact embedding,

- $B \subset Y$ with continuous embedding.

Let $T>0$ and $\left(u^{(n)}\right)_{n \in \mathbb{N}}$ be a sequence such that:

1. $\left(u^{(n)}\right)_{n \in \mathbb{N}}$ is bounded in $\mathrm{L}^{q}((0, T), X)$,

2. $\left(\frac{d u^{(n)}}{d t}\right)_{n \in \mathbb{N}}$ is bounded in $\mathrm{L}^{q}((0, T), Y)$.

Then there exists $u \in \mathrm{L}^{q}((0, T), B)$ such that, up to a subsequence, $u^{(n)} \rightarrow u$ in $\mathrm{L}^{q}((0, T), B)$. 
Proof. The proof uses Theorem 2.1. Let $\varphi \in \mathrm{C}_{c}^{\infty}((-T, 2 T), \mathbb{R})$ such that $0 \leq \varphi \leq 1$ in $(-T, 2 T)$ and $\varphi=1$ in $[0, T]$. For $n \in \mathbb{N}$, we define $\bar{u}^{(n)}$ and $P u^{(n)}$ as follow:

$$
\begin{array}{r}
\bar{u}^{(n)}(t)=u^{(n)}(t) \text { for } t \in[0, T], \\
\bar{u}^{(n)}(t)=u^{(n)}(-t) \text { for } t \in(-T, 0), \\
\bar{u}^{(n)}(T+t)=u^{(n)}(T-t) \text { for } t \in(0, T) .
\end{array}
$$

and $P u^{(n)}(t)=\varphi(t) \bar{u}^{(n)}(t)$ for all $t \in(-T, 2 T)$ (and for all $t \in \mathbb{R}$ using $\varphi(t)=0$ if $t \notin(-T, 2 T))$.

We now prove that the three hypotheses of Theorem 2.1 are satisfied for the set $A=\left\{u^{(n)}, n \in \mathbb{N}\right\}$ (and this will conclude the proof of Theorem 2.2).

Hypothesis 1 of Theorem 2.1. Since the sequence $\left(u^{(n)}\right)_{n \in \mathbb{N}}$ is bounded in $\mathrm{L}^{q}((0, T), X)$, it is also bounded in $\mathrm{L}^{q}((0, T), B)$ (because $X$ is continuously embedded in $B)$. Therefore, the hypothesis 1 of Theorem 2.1 is clearly satisfied since $P u^{(n)}=u^{(n)}$ a.e. in $(0, T)$ and $\left\|P u^{(n)}\right\|_{L^{q}(\mathbb{R}, B)}^{q} \leq 3\left\|u^{(n)}\right\|_{L^{q}((0, T), B)}^{q}$.

Hypothesis 2 of Theorem 2.1. Let $\psi \in \mathrm{C}_{c}^{\infty}(\mathbb{R}, \mathbb{R})$. We have, using the notation $\|\psi\|_{u}=\max _{t \in \mathbb{R}}|\psi(t)|$,

$$
\begin{aligned}
\left\|\int_{\mathbb{R}} P u^{(n)}(t) \psi(t) \mathrm{d} t\right\|_{X} & \leq\|\psi\|_{u}\left\|P u^{(n)}\right\|_{L^{1}(\mathbb{R}, X)} \\
& \leq(3 T)^{1-1 / q}\|\psi\|_{u}\left\|P u^{(n)}\right\|_{L^{q}(\mathbb{R}, X)} .
\end{aligned}
$$

Since $\left(u^{(n)}\right)_{n \in \mathbb{N}}$ is bounded in $\mathrm{L}^{q}((0, T), X)$, the sequence $\left(P u^{(n)}\right)_{n \in \mathbb{N}}$ is bounded in $\mathrm{L}^{q}(\mathbb{R}, X)$. Then (2) gives that the sequence $\left\{\int_{\mathbb{R}} P u^{(n)}(t) \psi(t) \mathrm{d} t, n \in \mathbb{N}\right\}$ is bounded in $X$. Since $X$ is compactly embedded in $B$, we conclude that the sequence $\left\{\int_{\mathbb{R}} P u^{(n)}(t) \psi(t) \mathrm{d} t, n \in \mathbb{N}\right\}$ is relatively compact in $B$.

Hypothesis 3 of Theorem 2.1. We have to prove that

$$
\left\|P u^{(n)}(\cdot+h)-P u^{(n)}\right\|_{\mathrm{L}^{q}(\mathbb{R}, B)} \rightarrow 0 \text { as } h \rightarrow 0 \text {, uniformly w.r.t. } n \in \mathbb{N} .
$$

Let $\delta>0$ such that $\varphi(t)=0$ if $t \notin(-T+\delta, 2 T-\delta)$. Without loss of generality, we may assume that $0<h<\delta$ for proving Assertion (3). For $0<h<\delta$ and $t \in(-T, 2 T-\delta)$, we have:

$P u^{(n)}(t+h)-P u^{(n)}(t)=(\varphi(t+h)-\varphi(t)) \bar{u}^{(n)}(t+h)+\varphi(t)\left(\bar{u}^{(n)}(t+h)-\bar{u}^{(n)}(t)\right)$.

Then, with $\left\|\varphi^{\prime}\right\|_{u}=\max _{s \in \mathbb{R}}\left|\varphi^{\prime}(s)\right|$,

$\left\|P u^{(n)}(t+h)-P u^{(n)}(t)\right\|_{B} \leq h\left\|\varphi^{\prime}\right\|_{u}\left\|\bar{u}^{(n)}(t+h)\right\|_{B}+\|\varphi\|_{u}\left\|\bar{u}^{(n)}(t+h)-\bar{u}^{(n)}(t)\right\|_{B}$.

We now use the Lions lemma 2.3 given below. For all $\epsilon>0$, we have:

$$
\begin{aligned}
& \left\|P u^{(n)}(t+h)-P u^{(n)}(t)\right\|_{B} \leq h\left\|\varphi^{\prime}\right\|_{u}\left\|\bar{u}^{(n)}(t+h)\right\|_{B} \\
& \quad+\epsilon\|\varphi\|_{u}\left\|\bar{u}^{(n)}(t+h)-\bar{u}^{(n)}(t)\right\|_{X}+C_{\epsilon}\|\varphi\|_{u}\left\|\bar{u}^{(n)}(t+h)-\bar{u}^{(n)}(t)\right\|_{Y},
\end{aligned}
$$

which gives also

$$
\begin{array}{r}
\left\|P u^{(n)}(t+h)-P u^{(n)}(t)\right\|_{B} \leq h\left\|\varphi^{\prime}\right\|_{u}\left\|\bar{u}^{(n)}(t+h)\right\|_{B}+\epsilon\|\varphi\|_{u}\left\|\bar{u}^{(n)}(t+h)\right\|_{X} \\
+\epsilon\|\varphi\|_{u}\left\|\bar{u}^{(n)}(t)\right\|_{X}+C_{\epsilon}\|\varphi\|_{u}\left\|\bar{u}^{(n)}(t+h)-\bar{u}^{(n)}(t)\right\|_{Y},
\end{array}
$$


and then, taking this inequality at the power $q$ and integrating with respect to $t$,

$$
\begin{aligned}
\| P u^{(n)}(\cdot+h)-P u^{(n)} & \|_{\mathrm{L}^{q}(\mathbb{R}, B)}^{q} \leq 3\left(4 h\left\|\varphi^{\prime}\right\|_{u}\left\|u^{(n)}\right\|_{\mathrm{L}^{q}((0, T), B)}\right)^{q} \\
& +3\left(8 \epsilon\|\varphi\|_{u}\left\|u^{(n)}\right\|_{\mathrm{L}^{q}((0, T), X)}\right)^{q} \\
& +\left(4 C_{\epsilon}\|\varphi\| u\right)^{q} \int_{-T}^{2 T-\delta}\left\|u^{(n)}(t+h)-u^{(n)}(t)\right\|_{Y}^{q} \mathrm{~d} t .
\end{aligned}
$$

We now use the second item of Remark 3. It gives that $u^{(n)} \in \mathrm{C}([0, T], Y)$ and

$$
u^{(n)}\left(t_{1}\right)-u^{(n)}\left(t_{2}\right)=\int_{t_{1}}^{t_{2}} \frac{d u^{(n)}}{d t}(s) \mathrm{d} s
$$

for all $t_{1}, t_{2} \in[0, T]$. Setting

$$
\begin{array}{r}
v^{(n)}(t)=\frac{d u^{(n)}}{d t}(t) \text { if } t \in(0, T), \\
v^{(n)}(t)=-\frac{d u^{(n)}}{d t}(-t) \text { if } t \in(-T, 0), \\
v^{(n)}(T+t)=-\frac{d u^{(n)}}{d t}(T-t) \text { if } t \in(0, T),
\end{array}
$$

we then have

$$
u^{(n)}\left(t_{1}\right)-u^{(n)}\left(t_{2}\right)=\int_{t_{1}}^{t_{2}} v(s) \mathrm{d} s \text { for all } t_{1}, t_{2} \in(-T, 2 T),
$$

and $\left\|v^{(n)}\right\|_{\mathrm{L}^{q}((-T, 2 T), Y)}^{q}=3\left\|\frac{d u^{(n)}}{d t}\right\|_{\mathrm{L}^{q}((0, T), Y)}^{q}$.

We are now able to bound the third term of the RHS of (4).

$$
\begin{array}{r}
\int_{-T}^{2 T-\delta}\left\|u^{(n)}(t+h)-u^{(n)}(t)\right\|_{Y}^{q} \mathrm{~d} t \leq \int_{-T}^{2 T-\delta}\left(\int_{t}^{t+h}\left\|v^{(n)}(s)\right\|_{Y} \mathrm{~d} s\right)^{q} \mathrm{~d} t \\
\leq \int_{-T}^{2 T-\delta} h^{1-1 / q}\left(\int_{-T}^{2 T} 1_{[t, t+h]}(s)\left\|v^{(n)}(s)\right\|_{Y}^{q} \mathrm{~d} s\right) \mathrm{d} t .
\end{array}
$$

Then, using $1_{[t, t+h]}(s)=1_{[s-h, s]}(t)$ and the Fubini-Tonelli theorem, we obtain

$$
\begin{aligned}
\int_{-T}^{2 T-\delta}\left\|u^{(n)}(t+h)-u^{(n)}(t)\right\|_{Y}^{q} \mathrm{~d} t & \leq h^{2-1 / q}\left\|v^{(n)}\right\|_{\mathrm{L}^{q}((-T, 2 T), Y)}^{q} \\
& =3 h^{2-1 / q}\left\|\frac{d u^{(n)}}{d t}\right\|_{\mathrm{L}^{q}((0, T), Y)}^{q}
\end{aligned}
$$

With (4) and (5), we can now conclude. Let $\eta>0$. There exists $h_{1} \in(0, \delta)$ such that the first term of the RHS of (4) is bounded by $\eta$ (independently of $n \in \mathbb{N}$ ) if $0<h<h_{1}$. For the second term of the RHS of (4), we choose $\epsilon>0$ in order to bound by $\eta$ this second term (independently of $n \in \mathbb{N}$ ). Now, since $C_{\epsilon}$ is given, thanks to (5), there exists $h_{2} \in(0, \delta)$ such that the third term of the RHS of (4) is bounded by $\eta$ (independently of $n \in \mathbb{N}$ ) if $0<h<h_{2}$. Finally, we obtain

$$
0<h<\min \left\{h_{1}, h_{2}\right\} \Rightarrow\left\|P u^{(n)}(\cdot+h)-P u^{(n)}\right\|_{\mathrm{L}^{q}(\mathbb{R}, B)}^{q} \leq 3 \eta .
$$

This concludes the proof of Theorem 2.2.

Remark 2. In the proof of Theorem 2.2, in the case where $\left(u^{(n)}\right)_{n \in \mathbb{N}}$ is bounded in $\mathrm{L}^{r}((0, T), B)$ for some $r>q$, it is quite simpler to define $P u^{(n)}$ taking $P u^{(n)}=0$ in $\mathbb{R} \backslash[0, T]$. 
Lemma 2.3. Let $X, B, Y$ be three Banach spaces such that:

$-X \subset B$ with compact embedding,

- $B \subset Y$ with continuous embedding.

Then, for any $\epsilon>0$, there exists $C_{\epsilon}$ such that, for $w \in X$,

$$
\|w\|_{B} \leq \epsilon\|w\|_{X}+C_{\epsilon}\|w\|_{Y} .
$$

Proof. We perform the proof by contradiction. We assume that there exists $\epsilon>0$ and a sequence $\left(u^{(n)}\right)_{n \in \mathbb{N}}$ such that $u^{(n)} \in X$ and $1=\left\|u^{(n)}\right\|_{B}>\epsilon\left\|u^{(n)}\right\|_{X}+$ $n\left\|u^{(n)}\right\|_{Y}$, for all $n \in \mathbb{N}$. Then, $\left(u^{(n)}\right)_{n \in \mathbb{N}}$ is bounded in $X$ and therefore relatively compact in $B$. Thus, we can assume that $u^{(n)} \rightarrow u$ in $B$ and $\|u\|_{B}=1$. Furthermore $u^{(n)} \rightarrow 0$ in $Y$ (since $\left.\left\|u^{(n)}\right\|_{Y} \leq 1 / n\right)$. Then the second hypothesis of the Lemma gives $u=0$, which is in contradiction with $\|u\|_{B}=1$.

Remark 3. We gather in this remark some comments on the assumptions of Theorem 2.2.

1. We begin with giving some precision on the sense of " $d u / d t \in \mathrm{L}^{q}((0, T), Y)$ ".

First, let us clarify the definition of the derivative. Let $q \in[0, \infty), X$ be a Banach space and $u \in \mathrm{L}^{q}((0, T), X)$. The weak derivative of $u$, denoted by $d u / d t$, is defined by its action on test functions, that is its action on $\varphi$ for all $\varphi \in \mathrm{C}_{c}^{\infty}((0, T))$ (note that $\varphi$ takes its values in $\mathbb{R}$, and admits a classical derivative denoted by $\left.\varphi^{\prime}\right)$, by the following relation:

$$
\left\langle\frac{d u}{d t}, \varphi\right\rangle=-\int_{0}^{T} \varphi^{\prime}(t) u(t) \mathrm{d} t .
$$

Note that the right-hand side has a precise meaning: actually, if $\varphi \in \mathrm{C}_{c}^{\infty}((0, T))$, the function $\varphi^{\prime} u$ belongs to $\mathrm{L}^{q}((0, T), X)$ and therefore to the space $\mathrm{L}^{1}((0, T), X)$, and, finally, $\langle d u / d t, \varphi\rangle \in X$.

Then, let $r \in[1, \infty)$ and $Y$ be another Banach space. Then $d u / d t \in \mathrm{L}^{r}((0, T), Y)$ means that there exists $v \in \mathrm{L}^{r}((0, T), Y)$ (and then $v$ is unique) such that:

$$
-\int_{0}^{T} \varphi^{\prime}(t) u(t) \mathrm{d} t=\int_{0}^{T} \varphi(t) v(t) \mathrm{d} t \text { for all } \varphi \in \mathrm{C}_{c}^{\infty}((0, T)) .
$$

But the above discussion yields that the left-hand side of this relation lies in $X$ while the right-hand side lies in $Y$; this equality thus makes sense only if both $X$ and $Y$ are included in a common space. This is the case here since, by assumption, $X \subset Y$. The assertion $d u / d t \in \mathrm{L}^{r}((0, T), Y)$ identifies $d u / d t$, which is a linear application on $\mathrm{C}_{c}^{\infty}((0, T))$ with values in $X$, with $v$ which belongs to $\mathrm{L}^{r}((0, T), Y)$.

2. If $u \in \mathrm{L}^{q}((0, T), X)$ and $d u / d t \in \mathrm{L}^{r}((0, T), Y)$, it is quite easy to prove that $u \in \mathrm{C}([0, T], Y)$ and that:

$$
u(t)=u(0)+\int_{0}^{t} \frac{d u}{d t}(s) \mathrm{d} s, \text { for all } t \in[0, T] .
$$

Indeed, let $v=d u / d t$ and define $w \in \mathrm{C}([0, T])$ by:

$$
w(t)=\int_{0}^{t} v(s) \mathrm{d} s \text { for all } t \in[0, T] .
$$

Let $t_{1}, t_{2} \in(0, T), t_{1}<t_{2}$. For $t \in(0, T)$ one sets, with $\rho^{(n)}$ defined by (1),

$$
\varphi^{(n)}(t)=\int_{0}^{t} \rho^{(n)}\left(t_{1}-s\right) \mathrm{d} s-\int_{0}^{t} \rho^{(n)}\left(t_{2}-s\right) \mathrm{d} s,
$$


so that, if $1 / n<t_{1}$ and $1 / n<T-t_{2}, \varphi^{(n)} \in \mathrm{C}_{c}^{\infty}((0, T), \mathbb{R})$ and $\left(\varphi^{(n)}\right)^{\prime}(t)=$ $\rho^{(n)}\left(t_{1}-t\right)-\rho^{(n)}\left(t_{2}-t\right)$ for $t \in(0, T)$. Then, we have:

$$
u \star \rho^{(n)}\left(t_{1}\right)-u \star \rho^{(n)}\left(t_{2}\right)=\int_{0}^{T} u(s)\left(\varphi^{(n)}\right)^{\prime}(s) \mathrm{d} s=-\int_{0}^{T} v(s) \varphi^{(n)}(s) \mathrm{d} s .
$$

One has (up to a subsequence) $u \star \rho^{(n)} \rightarrow u$ a.e. in $X$ and then in $Y$ (as $n \rightarrow+\infty$ ). Since $\varphi^{(n)}$ tends a.e. in $(0, T)$ to the characteristic function of $\left[t_{1}, t_{2}\right]$ (and takes its values between 0 and 1$)$ we have:

$$
-\int_{0}^{T} v(s) \varphi^{(n)}(s) \mathrm{d} s \rightarrow-\int_{t_{1}}^{t_{2}} v(s) \mathrm{d} s \text { in } Y .
$$

Then we conclude that, for a.e. $t_{1}$ and $t_{2}$ in $(0, T)$, one has:

$$
u\left(t_{1}\right)-u\left(t_{2}\right)=-\int_{t_{1}}^{t_{2}} v(s) \mathrm{d} s=w\left(t_{1}\right)-w\left(t_{2}\right) .
$$

This proves that there exists $c \in \mathbb{R}$ such that $u=w+c$ a.e. on $(0, T)$ and then that $u \in \mathrm{C}([0, T], Y)$ (since, as usual, we identify $u$ with the continuous function $w+c)$.

3. In Theorem 2.2, thanks to the previous item of the present remark, one has $u^{(n)} \in \mathrm{C}([0, T], Y)$ for all $n \in \mathbb{N}$. However, the limit $u$ is not necessarily continuous. A simple example is obtained with $q=1, X=B=Y=\mathbb{R}$ and, for instance, $T=2$. Then, it is quite easy to construct a sequence $\left(u^{(n)}\right)_{n \in \mathbb{N}}$ bounded in $\mathrm{W}^{1,1}((0, T))$ the limit of which is the characteristic function of $[1,2]$.

Remark 4. The hypotheses of Lemma 2.3, and the same hypotheses of Theorem 2.2 , can be replaced by the following ones (and this way is better for understanding the generalization given in the next section):

1. Let $X \subset B$. The hypothesis " $X$ compactly embedded in $B$ " is equivalent to the following assumption: if $\left(\left\|w^{(n)}\right\|_{X}\right)_{n \in \mathbb{N}}$ is bounded, then, up to a subsequence, there exists $w \in B$ such that $w^{(n)} \rightarrow w$ in $B$.

2. Let $B \subset Y$. The hypothesis " $B$ continuously embedded in $Y$ " may be replaced by the following assumption: if $w^{(n)} \rightarrow w$ in $B$ and $\left\|w^{(n)}\right\|_{Y} \rightarrow 0$, then $w=0$.

We can also remark that the hypothesis $B \subset Y$ is not necessary in Lemma 2.3 and Theorem 2.2.

Remark 5. In some cases, the estimate given by Lemma 2.3 and used in Theorem 2.2 can be obtained by the following alternative construction. Let $B$ be a Hilbert space and $X$ be a Banach space, with $X \subset B$. We define on $X$ the dual norm of $\|\cdot\|_{X}$, denoted by $\|\cdot\|_{Y}$, with respect to the inner product of $B$, by:

$$
\|u\|_{Y}=\sup \left\{(u / v)_{B}, v \in X,\|v\|_{X} \leq 1\right\} .
$$

Then, for any $\epsilon>0$ and $w \in X$,

$$
\|w\|_{B} \leq \epsilon\|w\|_{X}+\frac{1}{4 \epsilon}\|w\|_{Y} .
$$

The proof is simple:

$$
\|u\|_{B}=(u / u)_{B}^{\frac{1}{2}} \leq\left(\|u\|_{Y}\|u\|_{X}\right)^{\frac{1}{2}} \leq \epsilon\|w\|_{X}+\frac{1}{4 \epsilon}\|w\|_{Y} .
$$

Note that the compactness of $X$ in $B$ is not needed here (but, even in this simple case of Lemma 2.3, the compactness of $X$ in $B$ is needed for Theorem 2.2.) 


\section{A discrete version of Lions and Aubin-Simon Lemmas.}

Lemma 3.1 (A discrete Lions Lemma). Let $\left(B^{(n)}\right)_{n \in \mathbb{N}}$ be a sequence of finitedimensional subspaces of a Banach space $B$ of norm $\|\cdot\|_{B}$. For $n \in \mathbb{N}$, let $\|\cdot\|_{X^{(n)}}$ and $\|\cdot\|_{Y^{(n)}}$ be two norms defined on the space $B^{(n)}$. We suppose that:

(H1) if $\left(u^{(n)}\right)_{n \in \mathbb{N}}$ is a sequence such that:

$$
\forall n \in \mathbb{N}, u^{(n)} \in B^{(n)} \text { and }\left\|u^{(n)}\right\|_{X^{(n)}} \leq C,
$$

with $C$ a constant real number, then, up to the extraction of a subsequence, $\left(u^{(n)}\right)_{n \in \mathbb{N}}$ converges to a limit $u \in B$ when $n$ tends to $+\infty$.

(H2) if the sequence $\left(u^{(n)}\right)_{n \in \mathbb{N}}$ converges to $u$ in $B$ when $n$ tends to $+\infty$, and $\lim _{n \rightarrow+\infty}\left\|u^{(n)}\right\|_{Y(n)}=0$, then $u=0$.

Then, for any $\epsilon>0$, there exists a real number $C(\epsilon)$ such that:

$$
\forall n \in \mathbb{N}, \forall u \in B^{(n)}, \quad\|u\|_{B} \leq \epsilon\|u\|_{X^{(n)}}+C(\epsilon)\|u\|_{Y^{(n)}} .
$$

Proof. For a fixed $n \in \mathbb{N}$, a relation of the form of Equation (6) is satisfied, since the dimension of $B^{(n)}$ is finite; the problem is thus only to prove that Equation (6) holds with $C(\epsilon)$ independent of $n$. This proof is performed by contradiction. If the Lemma is false, it means that it is possible to find $\epsilon_{0}>0$ and to build a sequence $\left(u^{(m)}\right)_{m \in \mathbb{N}}$ such that, $\forall m \in \mathbb{N}$, there exists $n_{m}$ such that $u^{(m)} \in B^{\left(n_{m}\right)}$ and:

$$
\left\|u^{(m)}\right\|_{B}>\epsilon_{0}\left\|u^{(m)}\right\|_{X^{\left(n_{m}\right)}}+m\left\|u^{(m)}\right\|_{Y^{\left(n_{m}\right)}}
$$

with $n_{m}$ tending to $+\infty$ with $m$. Let us consider the sequence $\left(v^{(m)}\right)_{m \in \mathbb{N}}$ defined by:

$$
\forall m \in \mathbb{N}, \quad v^{(m)}=\frac{1}{\left\|u^{(m)}\right\|_{B}} u^{(m)} \quad\left(\text { thus }\left\|v^{(m)}\right\|_{B}=1\right) .
$$

By (7), we know that:

$$
\forall m \in \mathbb{N}, \quad\left\|v^{(m)}\right\|_{X^{\left(n_{m}\right)}} \leq \frac{1}{\epsilon_{0}} .
$$

By Assumption (H1), a subsequence of $\left(v^{(m)}\right)_{m \in \mathbb{N}}$, still denoted by $\left(v^{(m)}\right)_{m \in \mathbb{N}}$, thus converges in $B$ to a limit $v$, which satisfies $\|v\|_{B}=1$. But, once again by (7), we get:

$$
\forall m \in \mathbb{N}, \quad\left\|v^{(m)}\right\|_{Y^{\left(n_{m}\right)}} \leq \frac{1}{m}
$$

and thus, by Assumption (H2), $v=0$, which is in contradiction with the fact that $\left\|v^{(m)}\right\|_{B}=1$.

We will need hereafter the following elementary lemma.

Lemma 3.2. The assumption (H1) of Lemma 3.1 yields that there exists $C_{X}$ such that:

$$
\forall n \in \mathbb{N}, \forall u \in B^{(n)}, \quad\|u\|_{B} \leq C_{X}\|u\|_{X^{(n)}}
$$

Proof. As in Lemma 3.1, if this assertion is false, there exists a sequence $\left(u^{(n)}\right)_{n \in \mathbb{N}}$ satisfying: $\forall n$, there exists $m_{n}$ such that $u^{(n)} \in B^{\left(m_{n}\right)},\left\|u^{(n)}\right\|_{X^{\left(m_{n}\right)}}=1$ and $\left\|u^{(n)}\right\|_{B} \geq n$, with $m_{n}$ tending to $+\infty$ with $n$. By assumption (H1), the sequence $\left(u^{(n)}\right)_{n \in \mathbb{N}}$ converges, up to the extraction of a subsequence, to a limit in $B$, which is in contradiction with the fact that $\left\|u^{(n)}\right\|_{B}$ blows up when $n \rightarrow \infty$. 
Definition 3.3. Let $T>0$ be given. For $n \in \mathbb{N}$, we suppose given a uniform partition of $[0, T)$, such that:

$$
[0, T)=\cup_{1 \leq i \leq N^{(n)}}\left[t_{i-1}^{(n)}, t_{i}^{(n)}\right), \quad\left(\text { so } t_{i}^{(n)}=i \delta t^{(n)}, \text { with } \delta t^{(n)}=T / N^{(n)}\right),
$$

and a finite dimensional Banach space $B^{(n)}$. Then, for $n \in \mathbb{N}$, the finite dimensional space $\mathrm{H}^{(n)}$ is defined as the space of piecewise constant functions over each $I_{i}^{(n)}=$ $\left(t_{i-1}^{(n)}, t_{i}^{(n)}\right)$, for $1 \leq i \leq N^{(n)}$ and taking their values in $B^{(n)}$. For any $u^{(n)} \in \mathrm{H}^{(n)}$ and $1 \leq i \leq N^{(n)}$, we denote by $u_{i}^{(n)}$ the value taken by $u^{(n)}$ on $I_{i}^{(n)}$. For $2 \leq i \leq$ $N^{(n)}$, we define $\partial_{t}^{(n)}\left(u^{(n)}\right)_{i} \in B^{(n)}$ by:

$$
\partial_{t}^{(n)}\left(u^{(n)}\right)_{i}=\frac{1}{\delta t^{(n)}}\left(u_{i}^{(n)}-u_{i-1}^{(n)}\right) .
$$

Theorem 3.4 (A discrete Aubin-Simon Lemma). Let $\left(u^{(n)}\right)_{n \in \mathbb{N}}$ be a sequence of functions such that, $\forall n \in \mathbb{N}, u^{(n)} \in \mathrm{H}^{(n)}$, where $\mathrm{H}^{(n)}$ is defined in Definition 3.3. Let $q \in[1,+\infty)$. Under the assumptions of Lemma 3.1, we suppose that there exists a real number $C_{\mathcal{F}}$ such that:

$$
\forall n \in \mathbb{N}, \quad \sum_{i=1}^{N^{(n)}} \delta t^{(n)}\left\|u_{i}^{(n)}\right\|_{X^{(n)}}^{q}+\sum_{i=2}^{N^{(n)}} \delta t^{(n)}\left\|\partial_{t}^{(n)}\left(u^{(n)}\right)_{i}\right\|_{Y^{(n)}}^{q} \leq C_{\mathcal{F}} .
$$

Then, up to the extraction of a subsequence, the sequence $\left(u^{(n)}\right)_{n \in \mathbb{N}}$ converges to a function $u$ in $\mathrm{L}^{q}((0, T), B)$.

Proof. The proof of this compactness result is obtained by applying Theorem 2.1. To this purpose, the first step is to extend the functions $\left(u^{(n)}\right)_{n \in \mathbb{N}}$ to $\mathrm{L}^{q}(\mathbb{R}, B)$. For $n \in \mathbb{N}$, we first define $\tilde{u}^{(n)}$ over $(-T, 2 T)$ by:

$$
\begin{aligned}
& \left(\tilde{u}^{(n)}\right)=\left(u^{(n)}\right)_{i} \quad \text { on }\left(-t_{i},-t_{i-1}\right), 1 \leq i \leq N^{(n)}, \\
& \left(\tilde{u}^{(n)}\right)=\left(u^{(n)}\right)_{i} \quad \text { on }\left(t_{i-1}, t_{i}\right)=I_{i}^{(n)}, 1 \leq i \leq N^{(n)}, \\
& \left(\tilde{u}^{(n)}\right)=\left(u^{(n)}\right)_{N^{(n)}+1-i}, \quad \text { on }\left(T+t_{i-1}, T+t_{i}\right), 1 \leq i \leq N^{(n)},
\end{aligned}
$$

Let now $\varphi$ be a function of $\mathrm{C}_{c}^{\infty}(\mathbb{R}, \mathbb{R})$, taking its value in $[0,1]$, and such that $\varphi=1$ on $[0, T]$ and $\varphi$ is equal to zero on $(-\infty,-T / 2) \cup(3 T / 2,+\infty)$. Without loss of generality for practical applications, we suppose that, $\forall n \in \mathbb{N}, N^{(n)}>1$, so that the intersection between the support of $\varphi$ and $\left(-T,-T+\delta t^{(n)}\right)$ or $\left(2 T-\delta t^{(n)}, 2 T\right)$ is $\emptyset$. We finally define the extension of $u^{(n)}$ to $\mathrm{L}^{1}(\mathbb{R}, B)$, denoted by $\bar{u}^{(n)}$, by:

$$
\bar{u}^{(n)}(t)=\varphi(t) \tilde{u}^{(n)}(t) .
$$

Note that the definition of $\bar{u}^{(n)}$ simply results from $\bar{u}^{(n)}(-t)=\varphi(-t) u^{(n)}(t)$, $\bar{u}^{(n)}(t)=\varphi(t) u^{(n)}(t)=u^{(n)}(t)$ and $\bar{u}^{(n)}(T+t)=\varphi(T+t) u^{(n)}(T-t)$ for $t \in(0, T)$.

Let us first prove that the $\|\cdot\|_{\mathrm{L}^{q}(\mathbb{R}, B)}$ norm of this extension is bounded independently of $n$. Since $\varphi \leq 1$, we get:

$$
\left\|\bar{u}^{(n)}\right\|_{\mathrm{L}^{q}(\mathbb{R}, B)}^{q} \leq 3 \sum_{i=1, N^{(n)}} \delta t^{(n)}\left\|u_{i}^{(n)}\right\|_{B}^{q}
$$

and thus, invoking Lemma 3.2, $\left\|\bar{u}^{(n)}\right\|_{\mathrm{L}^{q}(\mathbb{R}, B)}^{q} \leq 3 C_{X}^{q} C_{\mathcal{F}}$. 
We now turn to point 2 of Theorem 2.1. Let $\psi \in \mathrm{C}_{c}^{\infty}(\mathbb{R}, \mathbb{R})$. We have, $\forall n \in \mathbb{N}$ :

$$
\int_{\mathbb{R}} \bar{u}^{(n)} \psi \mathrm{d} t \in B^{(n)}, \quad \int_{\mathbb{R}} \bar{u}^{(n)} \psi \mathrm{d} t=\sum_{i=1}^{N^{(n)}} c_{i}^{\psi} \delta t^{(n)} u_{i}^{(n)},
$$

where the coefficients $c_{i}^{\psi}$ are the sum of three bounded quantities, being the mean value over an interval of length $\delta t^{(n)}$ respectively of $\psi$ for one of them and of $\psi \varphi$ for the other two ones. We thus have:

$$
\left\|\int_{\mathbb{R}} \bar{u}^{(n)} \psi \mathrm{d} t\right\|_{X^{(n)}} \leq C_{\psi \varphi} \sum_{i=1}^{N^{(n)}} \delta t^{(n)}\left\|u_{i}^{(n)}\right\|_{X^{(n)}},
$$

where $C_{\psi \varphi}$ only depends on $\psi$ and $\varphi$. This inequality yields an estimate of its lefthand side, using a discrete Hölder inequality (thanks to the fact that $T$ is finite) and invoking (9). By assumption $H 1$, the sequence $\left(\int_{\mathbb{R}} \bar{u}^{(n)} \psi \mathrm{d} t\right)_{n \in \mathbb{N}}$ is thus relatively compact in $B$, which is the result we are looking for.

The last step to complete the proof is to bound the translates of the functions of $\left(\bar{u}^{(n)}\right)_{n \in \mathbb{N}}$. Let $\epsilon>0$; we are going to show that there exists $\delta(\epsilon)$ such that, $\forall n \in \mathbb{N}$, if the real number $\tau$ satisfies $|\tau|<\delta(\epsilon)$, then:

$$
\left\|\bar{u}^{(n)}(\cdot+\tau)-\bar{u}^{(n)}(\cdot)\right\|_{\mathrm{L}^{q}(\mathbb{R}, B)} \leq \epsilon .
$$

Let $\tau \in \mathbb{R}$, which me may suppose positive (the bound for $\tau$ negative being obtained from the result with $\tau>0$ by a simple change of variable). We suppose in addition that $\tau<T / 2$. We have:

$$
\left\|\bar{u}^{(n)}(\cdot+\tau)-\bar{u}^{(n)}(\cdot)\right\|_{\mathrm{L}^{q}(\mathbb{R}, B)}=\left\|\varphi(\cdot+\tau) \tilde{u}^{(n)}(\cdot+\tau)-\varphi(\cdot) \tilde{u}^{(n)}(\cdot)\right\|_{\mathrm{L}^{q}(\mathbb{R}, B)},
$$

and we decompose this expression in $T_{1}+T_{2}$, with:

$$
\begin{aligned}
& T_{1}=\left\|[\varphi(\cdot+\tau)-\varphi(\cdot)] \tilde{u}^{(n)}(\cdot+\tau)\right\|_{\mathrm{L}^{q}((-T, 3 T / 2), B)}, \\
& T_{2}=\left\|\varphi(\cdot)\left[\tilde{u}^{(n)}(\cdot+\tau)-\tilde{u}^{(n)}(\cdot)\right]\right\|_{\mathrm{L}^{q}((-T / 2,3 T / 2), B)} .
\end{aligned}
$$

For the first term, the triangle inequality yields:

$$
\begin{aligned}
\left|T_{1}\right| & \leq\|\varphi\|_{\mathrm{W}^{1, \infty}(\mathbb{R})} \tau\left\|\tilde{u}^{(n)}(\cdot+\tau)\right\|_{\mathrm{L}^{q}((-T, 3 T / 2), B)} \\
& \leq\|\varphi\|_{\mathrm{W}^{1, \infty}(\mathbb{R})} \tau\left(3 \sum_{i=1}^{N^{(n)}} \delta t^{(n)}\left\|u_{i}^{(n)}\right\|_{B}^{q}\right)^{1 / q}
\end{aligned}
$$

and thus $\left|T_{1}\right| \leq C_{\varphi} \tau$, where $C_{\varphi}$ only depends on $\varphi, C_{X}$ in Lemma 3.2 and $C_{\mathcal{F}}$. Let us now turn to $T_{2}$. Since $\varphi \leq 1$, we get:

$$
\left|T_{2}\right|^{q} \leq \int_{-T}^{2 T}\left\|\tilde{u}^{(n)}(t+\tau)-\tilde{u}^{(n)}(t)\right\|_{B}^{q} \mathrm{~d} t .
$$

Applying Lemma 3.1, we get that there exists a real number $C_{\epsilon}$ depending on $\epsilon, q$ and $C_{\mathcal{F}}$ but not on $n$ through Lemma 3.1 such that:

$$
\begin{aligned}
\left|T_{2}\right|^{q} & \leq \frac{\epsilon^{q}}{2^{2 q} 6 C_{\mathcal{F}}} \int_{-T}^{2 T}\left\|\tilde{u}^{(n)}(t+\tau)-\tilde{u}^{(n)}(t)\right\|_{X^{(n)}}^{q} \mathrm{~d} t \\
& +C_{\epsilon} \int_{-T}^{2 T}\left\|\tilde{u}^{(n)}(t+\tau)-\tilde{u}^{(n)}(t)\right\|_{Y^{(n)}}^{q} \mathrm{~d} t .
\end{aligned}
$$


By the triangle inequality, the first term at the right-hand side satisfies:

$$
T_{2,1} \leq \frac{\epsilon^{q}}{2^{2 q} 6 C_{\mathcal{F}}} \int_{-T}^{2 T}\left(\left\|\tilde{u}^{(n)}(t+\tau)\right\|_{X^{(n)}}^{q}+\left\|\tilde{u}^{(n)}(t)\right\|_{X^{(n)}}^{q}\right) \mathrm{d} t \leq\left(\frac{\epsilon}{2}\right)^{q} .
$$

For $-N^{(n)} \leq i \leq 2 N^{(n)}$, let us define $t_{i}=i \delta t^{(n)}$ and let us denote by $\left[\tilde{u}^{(n)}\right]_{i}$ the jump of $\tilde{u}^{(n)}$ at $t_{i}$. Let $\chi_{i}^{\tau}: \mathbb{R} \rightarrow \mathbb{R}$ be the function defined by $\chi_{i}^{\tau}(t)=1$ if $t<t_{i}<t+\tau$ and $\chi_{i}^{\tau}(t)=0$ otherwise. Then, for a.e. $t \in(-T, 2 T)$, the difference $\tilde{u}(t+\tau)-\tilde{u}(t)$ can be expanded as follows:

$$
\tilde{u}(t+\tau)-\tilde{u}(t)=\sum_{i>-N^{(n)}}^{i<N^{(n)}} \chi_{i}^{\tau}(t)\left[\tilde{u}^{(n)}\right]_{i}
$$

In addition, the function $\chi_{i}^{\tau}$ is the characteristic function of the interval $\left(t_{i}-\tau, t_{i}\right)$ and thus, for $-N^{(n)} \leq i \leq 2 N^{(n)}$ :

$$
\int_{\mathbb{R}} \chi_{i}^{\tau}(t) \mathrm{d} t=\tau
$$

In addition, we have:

$$
\delta t^{(n)} \sum_{i>-N^{(n)}}^{i<N^{(n)}} \chi_{i}^{\tau}(t) \leq \tau+\delta t^{(n)}
$$

With these notations, we get for the second term in Relation (12):

$$
T_{2,2}=C_{\epsilon} \int_{-T}^{2 T}\left\|\sum_{i>-N^{(n)}}^{i<N^{(n)}} \chi_{i}^{\tau}(t)\left[\tilde{u}^{(n)}\right]_{i}\right\|_{Y^{(n)}}^{q} \mathrm{~d} t .
$$

and thus, remarking that, in the above relation, $\chi_{i}^{\tau}(t)$ (the value of which is 0 or 1 ) may be replaced by $\chi_{i}^{\tau}(t)^{2}$, the discrete Hölder inequality yields:

$$
T_{2,2} \leq C_{\epsilon} \sum_{i>-N^{(n)}}^{i<N^{(n)}} \delta t^{(n)}\left\|\frac{\left[\tilde{u}^{(n)}\right]_{i}}{\delta t^{(n)}}\right\|_{Y^{(n)}}^{q} \int_{-T}^{2 T} \chi_{i}^{\tau}(t)\left(\sum_{i>-N^{(n)}}^{i<N^{(n)}} \delta t^{(n)} \chi_{i}^{\tau}(t)\right)^{q-1} \mathrm{~d} t,
$$

which, invoking (14), (15) and the bound for the discrete derivative of $u^{(n)}$, yields:

$$
T_{2,2} \leq 3 C_{\epsilon} C_{\mathcal{F}} \tau\left(\tau+\delta t^{(n)}\right)^{q-1} .
$$

Gathering the estimates of $T_{1}, T_{2,1}$ and $T_{2,2}$, we conclude that if $\tau \leq \delta(\epsilon)$ with:

$$
C_{\varphi} \delta(\epsilon)+\left[3 C_{\epsilon} C_{\mathcal{F}} \delta(\epsilon)(2 T)^{q-1}\right]^{1 / q} \leq \frac{\epsilon}{2}, \quad \delta(\epsilon) \leq T / 2,
$$

we obtain the desired (independent of $n$ ) bound for the translates of $\bar{u}^{(n)}$, which concludes the proof.

Remark 6. In the statement of Theorem 3.4, we can replace the assumption (H2) of Lemma 3.1 by the following one:

(H'2) The norms $\|\cdot\|_{X^{(n)}}$ and $\|\cdot\|_{Y^{(n)}}$ are dual with respect to the $\|\cdot\|_{B}$ norm.

Note that assumption (H'2) is sufficient by itself to obtain an estimate similar to the result of Lemma 3.1. However, the compactness assumption (H1) is used in the proof of Theorem 3.4. 
4. Application to a reduced turbulence model. In this section, we apply the abstract result of Theorem 3.4 to prove the convergence of a discretization of a model problem issued from the so-called Reynolds Average Modelling (RANS) of turbulent flows. Precisely speaking, the problem we address here reads:

$$
\begin{aligned}
\partial_{t} \boldsymbol{u}-\operatorname{div}(\mu(k) \nabla \boldsymbol{u})+\boldsymbol{\nabla} p & =\boldsymbol{f}, \\
\operatorname{div} \boldsymbol{u} & =0, \\
\partial_{t} k-\operatorname{div}(\mu(k) \nabla k)+\operatorname{div}(k \boldsymbol{u}) & =\mu_{t}(k)|\boldsymbol{\nabla} \boldsymbol{u}|^{2},
\end{aligned}
$$

where $\boldsymbol{u}$ and $p$ stands for the (Reynolds-averaged) vector-valued velocity and scalar pressure fields, respectively, $k$ stands for a scalar quantity which, from a physical point of view, is often identified to the turbulent kinetic energy (i.e. the kinetic energy associated to the fluctuations of the velocity), and $\boldsymbol{f}$ is a known forcing term. The problem is posed over $Q=\Omega \times(0, T)$, where $\Omega$ is a bounded domain of $\mathbb{R}^{d}$, $d=2$ or $d=3$, supposed to be polygonal $(d=2)$ or polyhedral $(d=3)$ and $T$ is the (finite) final time. For the sake of simplicity, we assume that $\boldsymbol{f} \in \mathrm{L}^{2}(Q)^{d}$ and we prescribe an homegeneous Dirichlet boundary condition for $\boldsymbol{u}$ and $k$, i.e.:

$$
\boldsymbol{u}(\boldsymbol{x}, t)=0, k(\boldsymbol{x}, t)=0, \text { for }(\boldsymbol{x}, t) \in \partial \Omega \times(0, T),
$$

where $\partial \Omega$ stands for the boundary of $\Omega$. The initial conditions are:

$$
\boldsymbol{u}(\boldsymbol{x}, 0)=\boldsymbol{u}_{\text {ini }}(\boldsymbol{x}), k(\boldsymbol{x}, 0)=k_{\text {ini }}(\boldsymbol{x}) \text { for a.e. } \boldsymbol{x} \in \Omega,
$$

with $\boldsymbol{u}_{\text {ini }} \in \mathrm{L}^{2}(\Omega)^{d}, k_{\text {ini }} \in \mathrm{L}^{1}(\Omega)$, and $\operatorname{div} \boldsymbol{u}_{\text {ini }}=0$ a.e. in $\Omega$. The diffusivity $\mu(k)$ is supposed to be given by:

$$
\mu(k)=\mu_{0}+\mu_{t}(k), \quad \mu_{t}(k)=\min \left[k^{1 / 2}, \mu_{t, \infty}\right]
$$

where $\mu_{0}>0$ and $\mu_{t, \infty}>0$, which is reminiscent of the so-called Prandtl model.

Since the usual energy identity associated to the unsteady Stokes problem (16)(17) yields a control of $\boldsymbol{u}$ in $\mathrm{L}^{2}\left((0, T), \mathrm{H}_{0}^{1}(\Omega)^{d}\right)$, the right-hand side of the balance equation for the turbulent energy, namely (18), only lies in $\mathrm{L}^{1}(Q)$.

The weak formulation of (16)-(18) may be written as follows:

Find $\boldsymbol{u} \in \mathrm{L}^{2}((0, T), \boldsymbol{V}) \cap \mathrm{L}^{\infty}\left((0, T), \mathrm{L}^{2}(\Omega)^{d}\right)$

and $k \in \mathrm{L}^{q}\left((0, T), \mathrm{W}_{0}^{1, q}(\Omega)\right)$, for any $q \in[1,(d+2) /(d+1))$

such that, $\forall \boldsymbol{\varphi} \in \boldsymbol{V} \cap \mathrm{C}_{c}^{\infty}(\Omega \times[0, T))^{d}$ and $\varphi \in \mathrm{C}_{c}^{\infty}(\Omega \times[0, T))$ :

$$
\begin{gathered}
-\int_{Q} \boldsymbol{u} \cdot \partial_{t} \boldsymbol{\varphi} \mathrm{d} \boldsymbol{x}+\int_{Q} \mu(k) \boldsymbol{\nabla} \boldsymbol{u}: \boldsymbol{\nabla} \boldsymbol{\varphi} \mathrm{d} \boldsymbol{x} \mathrm{d} t \\
=\int_{Q} \boldsymbol{f} \cdot \boldsymbol{\varphi} \mathrm{d} \boldsymbol{x} \mathrm{d} t+\int_{\Omega} \boldsymbol{u}_{\mathrm{ini}} \cdot \boldsymbol{\varphi}(\boldsymbol{x}, 0) \mathrm{d} \boldsymbol{x}, \\
-\int_{Q} k \partial_{t} \varphi \mathrm{d} \boldsymbol{x} \mathrm{d} t+\int_{Q} \mu(k) \boldsymbol{\nabla} k \cdot \boldsymbol{\nabla} \varphi \mathrm{d} \boldsymbol{x} \mathrm{d} t-\int_{Q} k \boldsymbol{u} \cdot \boldsymbol{\nabla} \varphi \mathrm{d} \boldsymbol{x} \mathrm{d} t \\
=\int_{Q} \mu_{t}(k)|\boldsymbol{\nabla} \boldsymbol{u}|^{2} \varphi \mathrm{d} \boldsymbol{x} \mathrm{d} t+\int_{\Omega} k_{\text {in }} \varphi(\boldsymbol{x}, 0) \mathrm{d} \boldsymbol{x},
\end{gathered}
$$

where the functional space $\boldsymbol{V}$ is defined by:

$$
\boldsymbol{V}=\left\{\boldsymbol{v} \in \mathrm{H}_{0}^{1}(\Omega)^{d} \text { s.t. } \operatorname{div} \boldsymbol{v}=0 \text { a.e. in } \Omega\right\} .
$$

For the mathematical analysis of a single elliptic or parabolic equation with $\mathrm{L}^{1}$ (or measure) right-hand side, we refer to $[2,1]$. Extension to a coupled system of 
two parabolic equations, the right-hand side of the second one being the energy associated to the first one, may be found in $[4,5]$. Finally, the existence of a weak solution to System (16)-(18) is proven in [12].

Our objective here is to propose a discretization for System (16)-(18) and show that numerical solutions converge to a weak solution of the problem, i.e. to a limit satisfying (21)-(22). The presentation is organized as follows. We first describe the space discretization (Section 4.1), then give the scheme (Section 4.2). Next step is to derive the estimates satisfied by the solution and prove its existence (Section 4.3). Then (Section 4.4), we check that the assumptions of Theorem 3.4 are satisfied, which yields the existence of a limit, the regularity of which is then made precise. Finally, we pass to the limit in the scheme (Section 4.5), to obtain that the limit indeed solves the weak continuous problem.

We restrict ourselves here to only sketch the main arguments of the proofs, the details being available in two recent papers, addressing the discretization, for the first one [11], of the steady counterpart of (16)-(18), and, for the second one [10], of a single parabolic equation with $\mathrm{L}^{1}$ right-hand side.

\subsection{Space discretization.}

4.1.1. The mesh and the time discretization. Let $\mathcal{M}$ be a partition of the domain $\Omega$ into disjoints simplices, supposed to be regular in the usual sense of the finite element literature (see e.g. [3]); in particular for any elements $K, L \in \mathcal{M}, \bar{K} \cap \bar{L}$ is either reduced to $\emptyset$, a vertex, (for $d=3$ ) a segment, or a whole face. The set of the faces of the mesh is denoted by $\mathcal{E}, \mathcal{E}_{\text {ext }}$ stands for the set of faces included in the boundary of $\Omega$, and the set of internal faces $\mathcal{E} \backslash \mathcal{E}_{\text {ext }}$ is denoted by $\mathcal{E}_{\text {int }}$. For every element $K \in \mathcal{M}, \mathcal{E}(K)$ represents the set of faces of $K$. The internal face $\sigma \in \mathcal{E}_{\text {int }}$ separating the control volumes $K$ and $L$ is denoted by $\sigma=K \mid L$. By $|\cdot|$, we denote either the $d$-dimensional or $(d-1)$-dimensional Lebesgue measure, so $|K|$ and $|\sigma|$ represent respectively the measure of the element $K$ and the face $\sigma$.

Moreover, for the discretization of a diffusion term by the finite volume method, we suppose that there exists a family $\mathcal{P}=\left(x_{K}\right)_{K \in \mathcal{M}}$ of points of $\Omega$ such that $x_{K} \in K$ for all $K \in \mathcal{M}$ and, for any internal face $\sigma=K \mid L$, the straight line going through $x_{K}$ and $x_{L}$ is orthogonal to $\sigma$. For any control volume $K$ and face $\sigma$ of $K$, we denote by $d_{K, \sigma}$ the Euclidean distance between $x_{K}$ and $\sigma$ and by $\boldsymbol{n}_{K, \sigma}$ the unit vector normal to $\sigma$ outward from $K$. For any face $\sigma$, we define $d_{\sigma}=d_{K, \sigma}+d_{L, \sigma}$, if $\sigma$ separates the two control volumes $K$ and $L$ (in which case $d_{\sigma}$ is the Euclidean distance between $x_{K}$ and $x_{L}$ ) and $d_{\sigma}=d_{K, \sigma}$ if $\sigma$ is included in the boundary.

We measure the regularity of the mesh by the parameter $\theta_{\mathcal{M}}>0$ defined by:

$$
\theta_{\mathcal{M}}=\inf _{K \in \mathcal{M}}\left\{\frac{d_{K, \sigma}}{d_{\sigma}} ; \sigma \in \mathcal{E}(K)\right\} \cup\left\{\frac{d_{K, \sigma}}{\operatorname{diam}(K)} ; \sigma \in \mathcal{E}(K)\right\} .
$$

The size of the mesh, $h_{\mathcal{M}}$, is defined by:

$$
h_{\mathcal{M}}=\max _{K \in \mathcal{M}} \operatorname{diam}(K) .
$$

Consistently with the previous section, we split the time interval $(0, T)$ into a uniform partition of time step $\delta t$, denote by $N$ the total number of time steps and define $t_{i}=i \delta t, 0 \leq i \leq N\left(\right.$ so $\left.\delta t=T / N,[0, T]=\cup_{1 \leq i \leq N}\left[t_{i-1}, t_{i}\right]\right)$. 
Definition 4.1 (Regular sequence of discretizations).

A sequence $\left(\mathcal{M}^{(n)}, \delta t^{(n)}\right)_{n \in \mathbb{N}}$ of meshes of $\Omega$ and time steps is said regular if:

1. $h_{\mathcal{M}}^{(n)} \rightarrow 0$ as $n \rightarrow+\infty$

2. $\delta t^{(n)} \rightarrow 0$ as $n \rightarrow+\infty$,

3. there exists $\theta_{0}>0$ such that $\theta_{\mathcal{M}}^{(n)} \geq \theta_{0}, \forall n \in \mathbb{N}$, with $\theta_{\mathcal{M}}^{(n)}$ defined by (23).

4.1.2. Nonconforming finite elements. For the (lowest order) Crouzeix-Raviart element [6], the discrete space for each component of the velocity is included in the space of piecewise affine polynomials. The mean value of the jump across an internal face of any discrete function is required to vanish. As usual in the finite element framework, the Dirichlet boundary conditions are enforced by the choice of the approximation space, so, here, the mean value of the velocity is required to vanish over each external face of the mesh. We denote by $U_{h}$ the discrete space for each component of the velocity, and by $\boldsymbol{U}_{h}=\left(U_{h}\right)^{d}$ the approximation space for the velocity.

The pressure is approximated by the space $M_{h}$ of piecewise constant functions over $\mathcal{M}$ :

$$
M_{h}=\left\{p \in \mathrm{L}^{2}(\Omega):\left.p\right|_{K} \text { constant }, \forall K \in \mathcal{M}\right\}
$$

Since only the continuity of the integral over each face of the mesh is imposed, the functions of $U_{h}$ are discontinuous through each face; the discretization is thus nonconforming in $\mathrm{H}^{1}(\Omega)^{d}$. We then define, for $1 \leq \ell \leq d$ and $v \in U_{h}, \partial_{h, \ell} v$ as the function of $\mathrm{L}^{2}(\Omega)$ which is equal to the (piecewise constant) derivative of $v$ with respect to the $\ell^{t h}$ space variable almost everywhere. This notation allows to define the discrete gradient, denoted by $\nabla_{h}$, for both scalar and vector valued discrete functions and the discrete divergence of vector valued discrete functions, denoted by $\operatorname{div}_{h}$.

The Crouzeix-Raviart pair of approximation spaces for the velocity and the pressure is inf-sup stable, in the usual sense for piecewise $\mathrm{H}^{1}$ discrete velocities, i.e. there exists $C_{i s}>0$ (independent of $m$ for a regular sequence of discretizations) such that:

$$
\forall p \in M_{h}, \quad \sup _{\boldsymbol{v} \in \boldsymbol{U}_{h}} \frac{1}{\|\boldsymbol{v}\|_{1, b}} \int_{\Omega} p \operatorname{div}_{h} \boldsymbol{v} \mathrm{d} \boldsymbol{x} \geq C_{i s}\|p-\bar{p}\|_{\mathrm{L}^{2}(\Omega)},
$$

where $\bar{p}$ stands for the mean value of $p$ over $\Omega$ and $\|\cdot\|_{1, b}$ stands for the broken Sobolev $\mathrm{H}^{1}$ semi-norm, which is defined for any function $v \in U_{h}$ or $v \in \boldsymbol{U}_{h}$ by:

$$
\|v\|_{1, b}^{2}=\int_{\Omega}\left|\nabla_{h} v\right|^{2} \mathrm{~d} \boldsymbol{x} .
$$

This broken Sobolev semi-norm is known to control the $\mathrm{L}^{2}$-norm by an extended Poincaré inequality [15, proposition 4.13]:

$$
\forall v \in U_{h}, \quad\|v\|_{\mathrm{L}^{2}(\Omega)} \leq c_{p}(\Omega)\|v\|_{1, b},
$$

where $c_{\mathrm{p}}(\Omega)>0$ only depends on the domain $\Omega$. 
4.1.3. Finite volumes: discrete space and norms. For a finite $q \geq 1$, we define a discrete $\mathrm{W}_{0}^{1, q}$-norm on $M_{h}$, the space of piecewise constant functions over any element $K \in \mathcal{M}$, by:

$$
\begin{aligned}
\forall v \in M_{h}, \quad\|v\|_{1, q, \mathcal{M}}^{q}= & \left.\sum_{\begin{array}{c}
\sigma \in \mathcal{E}_{\mathrm{int}}, \sigma=K \mid L \\
\end{array}}\left|\sum_{\sigma \in \mathcal{E}_{\mathrm{ext}}, \sigma \in \mathcal{E}(K)}\right| \sigma\left|d_{\sigma}\right| \frac{v_{K}-v_{L}}{d_{\sigma}}\right|^{q}\left|\frac{v_{K}}{d_{\sigma}}\right|^{q} .
\end{aligned}
$$

We also define:

$$
\begin{aligned}
\|v\|_{1, \infty, \mathcal{M}}= & \max \left\{\left\{\frac{\left|v_{K}-v_{L}\right|}{d_{\sigma}}, \sigma \in \mathcal{E}_{\text {int }}, \sigma=K \mid L\right\}\right. \\
& \left.\cup\left\{\frac{\left|v_{K}\right|}{d_{\sigma}}, \sigma \in \mathcal{E}_{\text {ext }}, \sigma \in \mathcal{E}(K)\right\} \cup\left\{\left|v_{K}\right|, K \in \mathcal{M}\right\}\right\} .
\end{aligned}
$$

For $q>1$, we associate to these norms a dual norm with respect to the $\mathrm{L}^{2}$-inner product, denoted by $\|\cdot\|_{-1, q^{\prime}, \mathcal{M}}$ with $q^{\prime}$ given by $1 / q+1 / q^{\prime}=1$ if $q$ is finite and $q^{\prime}=1$ if $q=+\infty$, and defined by:

$$
\|v\|_{-1, q^{\prime}, \mathcal{M}}=\sup _{w \in M_{h}, w \neq 0} \frac{1}{\|w\|_{1, q, \mathcal{M}}} \int_{\Omega} v w \mathrm{~d} \boldsymbol{x} .
$$

4.2. The scheme. The considered numerical scheme for the discretization of Problem (16)-(18) combines a standard finite element discretization of the momentum balance equation and an upwind finite volume scheme for the equation satisfied by the turbulent scale $k$. The unknowns are thus:

$$
\left(\boldsymbol{u}_{i}\right)_{1 \leq i \leq N} \subset \boldsymbol{U}_{h},\left(p_{i}\right)_{1 \leq i \leq N} \subset M_{h},\left(k_{i}\right)_{1 \leq i \leq N} \subset M_{h},
$$

and are associated to functions of $\mathrm{L}^{\infty}(Q)$ defined by $\boldsymbol{u}(\boldsymbol{x}, t)=\boldsymbol{u}_{i}(\boldsymbol{x}), p(\boldsymbol{x}, t)=p_{i}(\boldsymbol{x})$, $k(\boldsymbol{x}, t)=k_{i}(\boldsymbol{x})$ for any $t \in\left(t_{i-1}, t_{i}\right), 1 \leq i \leq N$. For $1 \leq i \leq N$, they are solution to:

$$
\begin{aligned}
& \forall \boldsymbol{v} \in \boldsymbol{U}_{h}, \\
& \int_{\Omega} \partial_{t} \boldsymbol{u}_{i} \cdot \boldsymbol{v} \mathrm{d} \boldsymbol{x}+\int_{\Omega} \mu\left(k_{i-1}\right) \nabla_{h} \boldsymbol{u}_{i}: \nabla_{h} \boldsymbol{v} \mathrm{d} \boldsymbol{x} \\
& -\int_{\Omega} p_{i} \operatorname{div}_{h} \boldsymbol{v} \mathrm{d} \boldsymbol{x}=\int_{t_{i-1}}^{t_{i}} \int_{\Omega} \boldsymbol{f} \cdot \boldsymbol{v} \mathrm{d} \boldsymbol{x} \mathrm{d} t \\
& \forall q \in M_{h}, \quad \int_{\Omega} q \operatorname{div}_{h} \boldsymbol{u}_{i} \mathrm{~d} \boldsymbol{x}=0, \\
& \forall K \in \mathcal{M} \\
& |K|\left(\partial_{t} k\right)_{i, K}+\sum_{\sigma=K \mid L} \frac{|\sigma|}{d_{\sigma}} \mu\left(k_{i-1}\right)_{\sigma}\left(k_{i, K}-k_{i, L}\right)+\sum_{\sigma \in \mathcal{E}(K) \cap \mathcal{E}_{\text {ext }}} \frac{|\sigma|}{d_{\sigma}} \mu\left(k_{i-1}\right)_{\sigma} k_{i, K} \\
& +\sum_{\sigma=K \mid L}\left(\mathrm{v}_{i, K, \sigma}^{+} k_{i, K}-\mathrm{v}_{i, K, \sigma}^{-} k_{i, L}\right)=|K|\left[\mu_{t}\left(k_{i-1}\right)\left|\nabla \boldsymbol{u}_{i}\right|^{2}\right]_{K}, \\
& \int_{\Omega} p_{i} \mathrm{~d} \boldsymbol{x}=0
\end{aligned}
$$


where the discrete time derivatives are defined by (8). In the latter relation, $\mathrm{v}_{i, K, \sigma}$ approximates the flux of $\boldsymbol{u}$ across the internal face $K \mid L$ outward the element $K$, and is defined by:

$$
\mathrm{v}_{i, K, \sigma}=\int_{\sigma=K \mid L} \boldsymbol{u}_{i}(\boldsymbol{x}) \cdot \boldsymbol{n}_{K, \sigma} \mathrm{d} \gamma(\boldsymbol{x})
$$

$\mathrm{v}_{i, K, \sigma}^{+}=\max \left(\mathrm{v}_{i, K, \sigma}, 0\right)$ and $\mathrm{v}_{i, K, \sigma}^{-}=-\min \left(\mathrm{v}_{i, K, \sigma}, 0\right)\left(\right.$ so $\left._{i, K, \sigma}=\mathrm{v}_{i, K, \sigma}^{+}-\mathrm{v}_{i, K, \sigma}^{-}\right)$. The discretization of the source term in (31) is given by:

$$
\left[\mu_{t}(k)|\nabla \boldsymbol{u}|^{2}\right]_{K}=\frac{\mu_{t}\left(k_{K}\right)}{|K|} \int_{K}\left|\nabla_{h} \boldsymbol{u}\right|^{2} \mathrm{~d} \boldsymbol{x}
$$

and, for $\sigma \in \mathcal{E}, \mu(k)_{\sigma}$ stands for a reasonable approximation of the viscosity on $\sigma$, supposed to satisfy at each internal face $\sigma=K \mid L$ :

$$
\min \left[\mu\left(k_{K}\right), \mu\left(k_{L}\right)\right] \leq \mu(k)_{\sigma} \leq \max \left[\mu\left(k_{K}\right), \mu\left(k_{L}\right)\right] .
$$

On an external face to the control volume $K$, we just set $\mu(k)_{\sigma}=\mu\left(k_{K}\right)$ For instance, on $\sigma=K \mid L, \mu_{\sigma}$ may be defined as the arithmetic or harmonic mean value of $\mu\left(k_{K}\right)$ and $\mu\left(k_{L}\right)$. The scheme is initialized with:

$$
\boldsymbol{u}_{0}=\boldsymbol{\pi}_{h} \boldsymbol{u}_{\text {ini }}, \quad k_{i, K}=\frac{1}{|K|} \int_{K} k_{\text {ini }} \mathrm{d} \boldsymbol{x},
$$

where $\boldsymbol{\pi}_{h}$ is a projection operator stable from $\mathrm{L}^{2}(\Omega)^{d}$ to $\boldsymbol{U}_{h}$, in the sense that, for any function $\boldsymbol{v} \in \mathrm{L}^{2}(\Omega)^{d}$ and any mesh $\mathcal{M}$ such that $\theta_{\mathcal{M}} \geq \theta_{0}$ :

$$
\|\boldsymbol{\pi} \boldsymbol{v}\|_{\mathrm{L}^{2}(\Omega)^{d}} \leq C\|\boldsymbol{v}\|_{\mathrm{L}^{2}(\Omega)^{d}}
$$

where $C$ only depends on $\theta_{0}$. In addition, we require that, for a sequence of meshes $\mathcal{M}^{(n)}$, possibly regular (i.e. satisfying $\theta_{\mathcal{M}^{(n)}}>\theta_{0}>0$ ) and for a given function $\boldsymbol{v} \in \mathrm{L}^{2}(\Omega)^{d}$, the sequence $\left(\boldsymbol{\pi}^{(n)} \boldsymbol{v}\right)_{n \in \mathbb{N}}$ converges to $\boldsymbol{v}$ in $\mathrm{L}^{2}(\Omega)^{d}$. For instance, one may give to each degree of freedom of $\boldsymbol{u}_{0}$ the mean value of $\boldsymbol{u}_{\text {ini }}$ over the support of its shape function.

Finally, note that, since the velocity is piecewise affine and so, $\forall \boldsymbol{v} \in \boldsymbol{U}_{h}, \operatorname{div}_{h} \boldsymbol{v}$ is piecewise constant, Equation (30) is equivalent to $\operatorname{div}_{h} \boldsymbol{u}_{i}=0$.

4.3. Estimates. Let us define on $\boldsymbol{U}_{h}$ the following semi-norm:

$$
\forall \boldsymbol{v} \in \boldsymbol{U}_{h}, \quad|\boldsymbol{v}|_{-1, \boldsymbol{V}_{h}^{\prime}}=\sup _{\boldsymbol{w} \in \boldsymbol{V}_{h}} \frac{1}{\|\boldsymbol{w}\|_{1, b}} \int_{\Omega} \boldsymbol{v} \cdot \boldsymbol{w} \mathrm{d} \boldsymbol{x},
$$

where:

$$
\boldsymbol{V}_{h}=\left\{\boldsymbol{v} \in \boldsymbol{U}_{h} \text { s.t. } \operatorname{div}_{h} \boldsymbol{v}=0\right\} .
$$

The solution to the scheme satisfies the following stability estimate.

Lemma 4.2. Let $\theta_{0}>0$ and let $\mathcal{M}$ be a triangulation of the computational domain $\Omega$ such that $\theta_{\mathcal{M}} \geq \theta_{0}$, where $\theta_{\mathcal{M}}$ is defined by (23). Then there exists a unique solution $(\boldsymbol{u}, p, k)$ to the scheme (29)-(32), initialized with (34), and this solution satisfies the following estimate:

$$
\begin{aligned}
\left\|\boldsymbol{u}_{N}\right\|_{\mathrm{L}^{2}(\Omega)^{d}}^{2}+ & \sum_{i=1}^{N} \delta t\left\|\boldsymbol{u}_{i}\right\|_{1, b}^{2}+\sum_{i=1}^{N} \delta t\left|\partial_{t} \boldsymbol{u}_{i}\right|_{-1, V_{h}^{\prime}} \\
& +\left\|k_{N}\right\|_{\mathrm{L}^{1}(\Omega)}+\sum_{i=1}^{N} \delta t\left\|k_{i}\right\|_{1, q, \mathcal{M}}+\sum_{i=1}^{N} \delta t\left\|\partial_{t} k_{i}\right\|_{-1,1, \mathcal{M}} \leq C,
\end{aligned}
$$


for any $q \in[1,(d+2) /(d+1))$ and where $C$ only depends on $\Omega, \boldsymbol{f}, \boldsymbol{u}_{\mathrm{ini}}, k_{\mathrm{ini}}, \mu_{0}, q$ and $\theta_{0}$.

Proof. We only give a brief sketch of the proof. First, we remark that the condition (30), together with the fact that the pressure is piecewise constant, implies that the following "discrete mass balance" is verified:

$$
\text { for } 1 \leq i \leq N, \forall K \in \mathcal{M}, \quad \sum_{\sigma \in \mathcal{E}(K)} \mathrm{v}_{i, K, \sigma}=0 .
$$

This condition, together with the particular discretization used for (31) and the initialization of the scheme, which yields $k_{0}>0$, implies that $k_{i}>0$, for $1 \leq$ $i \leq N$. This is an attractive property of the Crouzeix-Raviart element (or, for quadrangles or hexahedra control volumes, of the Rannacher-Turek element [13]). As a consequence, the effective viscosity $\mu(k)$ is correctly defined.

Taking $\boldsymbol{v}=\boldsymbol{u}_{i}$ in the first equation of the scheme, invoking the fact that $\mu(s) \geq$ $\mu_{0}, \forall s \geq 0$ and the discrete Poincaré inequality (25) yields:

$$
\left\|\boldsymbol{u}_{N}\right\|_{\mathrm{L}^{2}(\Omega)^{d}}^{2}+\sum_{i=1}^{N} \delta t\left\|\boldsymbol{u}_{i}\right\|_{1, b}^{2} \leq C, \quad \sum_{i=1}^{N} \delta t \sum_{K \in \mathcal{M}}|K|\left[\mu_{t}\left(k_{i-1}\right)\left|\nabla \boldsymbol{u}_{i}\right|^{2}\right]_{K} \leq C,
$$

with $C$ only depending on the data, as stated in the lemma, and thus the right hand side of (31) is bounded in $\mathrm{L}^{1}(Q)$. The estimate for $k$ is then obtained by testing (31) against a nonlinear function of $k$ lying in $\mathrm{L}^{\infty}(Q)$; this (rather technical) proof involves in particular discrete Sobolev inequalities [7, 8], and may be found in [10]. Finally, the estimate of the discrete derivatives of $\boldsymbol{u}$ and $k$ are obtained by testing (29) and (31) against functions of $\boldsymbol{V}_{h}$ and $M_{h}$ respectively, and invoking the already proven bounds of the unknowns.

The fact that the solution exists and is unique follows from an additional estimate for the last unknown, namely $p$, given by the discrete inf-sup condition (24) combined with (32), and finally remarking that, at each time step, (31) and (29)-(30) are two decoupled blocks of equations which can be solved successively, and this leads to solve only linear systems.

\subsection{Compactness of sequences of solutions.}

Lemma 4.3. Let $\left(\mathcal{M}^{(n)}, \delta t^{(n)}\right)_{n \in \mathbb{N}}$ be a regular sequence of meshes of $\Omega$ and time steps, in the sense of Definition 4.1. Let $\left(\boldsymbol{u}^{(n)}, k^{(n)}\right)_{n \in \mathbb{N}}$ be the corresponding sequence of discrete solutions. Then, up to the extraction of a subsequence:

- the sequence $\left(\boldsymbol{u}^{(n)}\right)_{n \in \mathbb{N}}$ converges in $\mathrm{L}^{2}(Q)^{d}$ to a limit $\boldsymbol{u}$, this limit satisfies $\boldsymbol{u} \in \mathrm{L}^{2}\left((0, T), \mathrm{H}_{0}^{1}(\Omega)^{d}\right)$ and the sequence of discrete gradients $\left(\boldsymbol{\nabla}_{h} \boldsymbol{u}^{(n)}\right)_{n \in \mathbb{N}}$ weakly converges in $\mathrm{L}^{2}(Q)^{d \times d}$ to $\boldsymbol{\nabla} \boldsymbol{u}$,

- the sequence $\left(k^{(n)}\right)_{n \in \mathbb{N}}$ converges in $\mathrm{L}^{1}(Q)$ to a limit $k$, and this limit satisfies $k \in \mathrm{L}^{q}\left((0, T), \mathrm{W}_{0}^{1, q}(\Omega)\right)$ for any $q \in[1,(d+2) /(d+1))$.

The proof of this result follows, for the convergence, from estimates of Lemma 4.2 and on the application of Theorem 3.4, the assumptions of which we now check. Let us begin with the sequence of discrete velocities $\left(\boldsymbol{u}^{(n)}\right)_{n \in \mathbb{N}}$. The proof of the following lemma may be found in [9, Theorem 3.3].

Lemma 4.4. Let $\left(\mathcal{M}^{(n)}\right)_{n \in \mathbb{N}}$ be a regular sequence of meshes of $\Omega$, in the sense that $\forall n \in \mathbb{N}$, the parameter $\theta_{\mathcal{M}^{(n)}}$ defined by (23) satisfies $\theta_{\mathcal{M}^{(n)}}>\theta_{0}>0$. Let 
$\left(u^{(n)}\right)_{n \in \mathbb{N}}$ be a sequence of discrete functions, i.e. such that $\forall n \in \mathbb{N}, u^{(n)}$ belongs to the space of Crouzeix-Raviart functions $U_{h}^{(n)}$ associated to $\mathcal{M}^{(n)}$. Let us suppose that there exists $C$ such that:

$$
\forall n \in \mathbb{N}, \quad\left\|u^{(n)}\right\|_{1, b} \leq C .
$$

Then, up to the extraction of a subsequence, the sequence $\left(u^{(n)}\right)_{n \in \mathbb{N}}$ converges in $\mathrm{L}^{2}(\Omega)$.

Since, for the velocity, the sequence of the solutions $\left(\boldsymbol{u}^{(n)}\right)_{n \in \mathbb{N}}$ and of its discrete time derivative $\left(\partial_{t} \boldsymbol{u}^{(n)}\right)_{n \in \mathbb{N}}$ are controlled by Lemma 4.2 in dual norms with respect to the $\mathrm{L}^{2}$ inner product, this lemma is sufficient to conclude to the convergence, applying Theorem 3.4 with assumptions (H1) and (H'2).

We now turn to the convergence of the discrete turbulent energy $\left(k^{(n)}\right)_{n \in \mathbb{N}}$. The following lemma may be found in [8].

Lemma 4.5. Let $\left(\mathcal{M}^{(n)}\right)_{n \in \mathbb{N}}$ be a regular sequence of meshes of $\Omega$, in the sense that $\forall n \in \mathbb{N}$, the parameter $\theta_{\mathcal{M}^{(n)}}$ defined by (23) satisfies $\theta_{\mathcal{M}^{(n)}}>\theta_{0}>0$. Let $\left(k^{(n)}\right)_{n \in \mathbb{N}}$ be a sequence of discrete functions, i.e. such that $\forall n \in \mathbb{N}, k^{(n)}$ belongs to the space of piecewise constant functions $M_{h}^{(n)}$ associated to $\mathcal{M}^{(n)}$. Let us suppose that there exists $C$ such that:

$$
\forall n \in \mathbb{N}, \quad\left\|k^{(n)}\right\|_{1,1, \mathcal{M}} \leq C .
$$

Then, up to the extraction of a subsequence, the sequence $\left(k^{(n)}\right)_{n \in \mathbb{N}}$ converges in $\mathrm{L}^{1}(\Omega)$.

In addition, we have the following result.

Lemma 4.6. As in the previous lemma, let $\left(\mathcal{M}^{(n)}\right)_{n \in \mathbb{N}}$ be a regular sequence of meshes of $\Omega$ and $\left(k^{(n)}\right)_{n \in \mathbb{N}}$ be a sequence of discrete functions. Let us suppose that:

$$
\lim _{n \rightarrow+\infty}\left\|k^{(n)}\right\|_{-1,1, \mathcal{M}}=0
$$

and that the sequence $\left(k^{(n)}\right)_{n \in \mathbb{N}}$ converges to $k$ in $\mathrm{L}^{1}(\Omega)$. Then $k=0$.

Proof. Let $\varphi \in \mathrm{C}_{c}^{\infty}(\Omega)$. For $n \in \mathbb{N}$, we denote by $\pi^{(n)} \varphi$ the discrete function of $M_{h}^{(n)}$ defined by $\left(\pi^{(n)} \varphi\right)_{K}=\varphi\left(\boldsymbol{x}_{K}\right), \forall K \in \mathcal{M}^{(n)}$. By the definition of the $\|\cdot\|_{1, \infty, \mathcal{M}^{(n)}}$ norm (27), we have:

$$
\left\|\pi^{(n)} \varphi\right\|_{1, \infty, \mathcal{M}^{(n)}} \leq\|\varphi\|_{\mathrm{W}^{1, \infty}(\Omega)} .
$$

We thus get, for $n \in \mathbb{N}$ and any $v^{(n)} \in M_{h}^{(n)}$ :

$$
\begin{array}{r}
\left|\int v^{(n)} \pi^{(n)} \varphi \mathrm{d} \boldsymbol{x}\right| \leq\left\|v^{(n)}\right\|_{-1,1, \mathcal{M}^{(n)}}\left\|\pi^{(n)} \varphi\right\|_{1, \infty, \mathcal{M}^{(n)}} \\
\leq\left\|v^{(n)}\right\|_{-1,1, \mathcal{M}^{(n)}}\|\varphi\|_{\mathrm{W}^{1, \infty}(\Omega)} .
\end{array}
$$

Let us suppose that the sequence $\left(v^{(n)}\right)_{n \in \mathbb{N}}$ converges in $\mathrm{L}^{1}(\Omega)$ to $v$ and that the sequence $\left(\left\|v^{(n)}\right\|_{-1,1, \mathcal{M}^{(n)}}\right)_{n \in \mathbb{N}}$ tends to zero when $n \rightarrow+\infty$. Then, passing to the limit in the above relation, since $\pi^{(n)} \varphi$ converges to $\varphi$ in $\mathrm{L}^{\infty}(\Omega)$, we get:

$$
\int v \varphi \mathrm{d} \boldsymbol{x}=0
$$

which, since this relation is valid for any $\varphi \in \mathrm{C}_{c}^{\infty}(\Omega)$, yields $v=0$.. 
Combining Lemma 4.5, Lemma 4.6 and the estimates of Lemma 4.2 yields the assumptions of Theorem 3.4 we are looking for, and thus the convergence, up to the extraction of a subsequence, of the sequence of solutions $\left(k^{(n)}\right)_{n \in \mathbb{N}}$.

The regularity of the limit is, once again, a consequence of the estimates, which give a control on the space (discrete) derivatives of the solutions. The weak convergence of the gradient of the velocity may be obtained by (easily) extending to the time-dependent case a proof which may be found in [11, Lemma 4].

\subsection{Convergence.}

Theorem 4.7. Let $\left(\mathcal{M}^{(n)}, \delta t^{(n)}\right)_{n \in \mathbb{N}}$ be a regular sequence of meshes of $\Omega$ and time steps, in the sense of Definition 4.1. Let $\left(\boldsymbol{u}^{(n)}, k^{(n)}\right)_{n \in \mathbb{N}}$ be the corresponding sequence of discrete solutions. Then, up to the extraction of a subsequence:

- the sequence $\left(\boldsymbol{u}^{(n)}\right)_{n \in \mathbb{N}}$ converges in $\mathrm{L}^{2}(Q)^{d}$ to a limit $\boldsymbol{u}$, and this limit satisfies $\boldsymbol{u} \in \mathrm{L}^{2}\left((0, T), \mathrm{H}_{0}^{1}(\Omega)^{d}\right)$,

- the sequence $\left(k^{(n)}\right)_{n \in \mathbb{N}}$ converges in $\mathrm{L}^{1}(Q)$ to a limit $k$, and this limit satisfies $k \in \mathrm{L}^{q}\left((0, T), \mathrm{W}_{0}^{1, q}(\Omega)\right)$ for any $q \in[1,(d+2) /(d+1))$,

and the limit $(\boldsymbol{u}, k)$ is solution to the weak formulation of the problem (21)-(22).

Proof. We begin with a brief sketch of the proof.

The first step is to pass to the limit in the scheme to show, using classical arguments, that the limit $\boldsymbol{u}$ satisfies (21) and is divergence free (see e.g. [9] for such a study).

The second step (the most difficult one) is to show the strong convergence of the dissipation, i.e. the right hand side of equation (18) (which also yields the strong convergence of the discrete gradient of the velocity). We detail this part of the proof hereafter.

Finally, to conclude, one must show that $k$ satisfies (22), which is done with a proof similar to the convergence proof given in [10], with two additional difficulties: the first one lies in the fact that the diffusion coefficient depends on $k$, and is easily handled using the strong convergence of $k$ in $\mathrm{L}^{1}(Q)$ (which, since the function $\mu$ is continuous and bounded, implies the convergence of $\mu\left(k^{(n)}\right)$ to $\mu(k)$ first almost everywhere in $\Omega$ (up to the extraction of a sequence, but the limit is unique, so the whole sequence converges) and then, by Lebesgue dominated convergence theorem, in any $\left.\mathrm{L}^{q}(Q), q \in[1,+\infty)[11]\right)$; the second additional difficulty with respect to [10] is the weaker control on the velocity in the present context, which is however sufficient to pass to the limit (see once again [9] for a similar proof).

We detail now the proof of the strong convergence of the dissipation. Let us take $\boldsymbol{u}_{i}^{(n)}$ as test function in (29), and sum over $i$, to obtain, for $1 \leq j \leq N^{(n)}$ :

$$
\begin{aligned}
\frac{1}{2} \int_{\Omega}\left|\boldsymbol{u}_{j}^{(n)}\right|^{2} \mathrm{~d} \boldsymbol{x}+\sum_{i=1}^{j} \delta t^{(n)} \int_{\Omega} & \mu\left(k_{i-1}\right)\left|\nabla_{h} \boldsymbol{u}_{i}^{(n)}\right|^{2} \mathrm{~d} \boldsymbol{x} \\
& \leq \int_{0}^{t_{j}} \int_{\Omega} \boldsymbol{f} \cdot \boldsymbol{u}^{(n)} \mathrm{d} \boldsymbol{x} \mathrm{d} t+\frac{1}{2} \int_{\Omega}\left|\boldsymbol{u}_{0}\right|^{2} \mathrm{~d} \boldsymbol{x} .
\end{aligned}
$$


Multiplying by $\delta t^{(n)}$, summing this relation from $j=1$ to $N^{(n)}$ and reorganizing the sums, we obtain:

$$
\begin{aligned}
& \sum_{i=1}^{N^{(n)}}\left(T-(i-1) \delta t^{(n)}\right) \delta t^{(n)} \int_{\Omega} \mu\left(k_{i-1}\right)\left|\nabla_{h} \boldsymbol{u}_{i}^{(n)}\right|^{2} \mathrm{~d} \boldsymbol{x} \\
& \quad \leq-\frac{1}{2} \int_{Q}\left|\boldsymbol{u}^{(n)}\right|^{2} \mathrm{~d} \boldsymbol{x} \mathrm{d} t+\frac{T}{2} \int_{\Omega}\left|\boldsymbol{u}_{0}^{(n)}\right|^{2} \mathrm{~d} \boldsymbol{x}+\int_{Q}(T-t)^{(n)} \boldsymbol{f} \cdot \boldsymbol{u}^{(n)} \mathrm{d} \boldsymbol{x} \mathrm{d} t,
\end{aligned}
$$

where $(T-t)^{(n)}$ is a piecewise function of $t$ defined by $(T-t)^{(n)}=T-(i-1) \delta t^{(n)}$ for $t \in\left(t_{i-1}, t_{i}\right)$. Using this notation in the first term of this relation and passing to the limit when $n \rightarrow+\infty$ yields, using the known convergence properties of the sequence $\left(\boldsymbol{u}^{(n)}\right)_{n \in \mathbb{N}}$ :

$$
\begin{aligned}
\limsup _{n \rightarrow+\infty} \int_{Q}(T-t)^{(n)} \mu\left(k_{i-1}\right)\left|\nabla_{h} \boldsymbol{u}_{i}^{(n)}\right|^{2} \mathrm{~d} \boldsymbol{x} \mathrm{d} t \\
\quad \leq-\frac{1}{2} \int_{Q}|\boldsymbol{u}|^{2} \mathrm{~d} \boldsymbol{x} \mathrm{d} t+\frac{T}{2} \int_{\Omega}\left|\boldsymbol{u}_{0}\right|^{2} \mathrm{~d} \boldsymbol{x}+\int_{Q}(T-t) \boldsymbol{f} \cdot \boldsymbol{u} \mathrm{d} \boldsymbol{x} \mathrm{d} t .
\end{aligned}
$$

We now use the fact that the solution $\boldsymbol{u}$ satisfies the first relation (21) of the weak continuous problem. From the regularity of $\boldsymbol{u}$ and $\partial_{t} \boldsymbol{u}$, this problem may be written:

$$
\int_{\Omega} \partial_{t} \boldsymbol{u} \cdot \boldsymbol{v} \mathrm{d} \boldsymbol{x}+\int_{\Omega} \mu(k) \boldsymbol{\nabla} \boldsymbol{u}: \boldsymbol{\nabla} \boldsymbol{v} \mathrm{d} \boldsymbol{x}=\int_{\Omega} \boldsymbol{f} \cdot \boldsymbol{v} \mathrm{d} \boldsymbol{x}, \quad \forall t \in(0, T), \forall \boldsymbol{v} \in \boldsymbol{V},
$$

with the initial condition $\boldsymbol{u}(\cdot, 0)=\boldsymbol{u}_{\text {ini }}$. Taking $\boldsymbol{u}$ as test function in this relation, and integrating twice in time, first for $t \in(0, s)$ and then for $s \in(0, T)$ yields:

$$
\begin{aligned}
\int_{Q}(T-t) & \mu(k)|\nabla \boldsymbol{u}|^{2} \mathrm{~d} \boldsymbol{x} \mathrm{d} t= \\
& -\frac{1}{2} \int_{Q}|\boldsymbol{u}|^{2} \mathrm{~d} \boldsymbol{x} \mathrm{d} t+\frac{T}{2} \int_{\Omega}\left|\boldsymbol{u}_{0}\right|^{2} \mathrm{~d} \boldsymbol{x}+\int_{Q}(T-t) \boldsymbol{f} \cdot \boldsymbol{u} \mathrm{d} \boldsymbol{x} \mathrm{d} t .
\end{aligned}
$$

Comparing (35) and (36), we finally get:

$$
\limsup _{n \rightarrow+\infty} \int_{Q}(T-t)^{(n)} \mu\left(k_{i-1}\right)\left|\nabla_{h} \boldsymbol{u}_{i}^{(n)}\right|^{2} \mathrm{~d} \boldsymbol{x} \mathrm{d} t \leq \int_{Q}(T-t) \mu(k)|\nabla \boldsymbol{u}|^{2} \mathrm{~d} \boldsymbol{x} \mathrm{d} t .
$$

The sequence $\left((T-t)^{(n)} \mu\left(k^{(n)}\right)^{1 / 2} \nabla_{h} \boldsymbol{u}^{(n)}\right)_{n \in \mathbb{N}}$ is bounded in $\mathrm{L}^{2}(Q)$, and thus weakly converges, up to the extraction of a subsequence, to a limit. Moreover, since, as explained before, $\mu\left(k^{(n)}\right)$ converges to $\mu(k)$ in any $\mathrm{L}^{q}(Q), q \in[1,+\infty)$, we may easily prove that this weak limit is $(T-t) \mu(k)^{1 / 2} \boldsymbol{\nabla} \boldsymbol{u}$ (thus, since this limit is unique, the whole sequence weakly converges). Relation (37) thus yields the strong convergence of this sequence, which, in turn, yields the strong convergence of the sequence $\left.\mu\left(k^{(n)}\right)^{1 / 2} \boldsymbol{\nabla}_{h} \boldsymbol{u}^{(n)}\right)_{n \in \mathbb{N}}$ to $\mu(k)^{1 / 2} \boldsymbol{\nabla} \boldsymbol{u}$ in $\mathrm{L}^{2}(\Omega \times(0, T-\epsilon))$ with $\epsilon>0$ (since, on $(0, T-\epsilon)$, the quantity $T-t$ is bounded from below by $\epsilon$ ).

We now prove that, still on $\mathrm{L}^{2}(\Omega \times(0, T-\epsilon))$, both $\left(\mu_{t}\left(k^{(n)}\right)^{1 / 2} \nabla_{h} \boldsymbol{u}^{(n)}\right)_{n \in \mathbb{N}}$ and $\left(\nabla_{h} \boldsymbol{u}^{(n)}\right)_{n \in \mathbb{N}}$ converge to $\mu_{t}(k)^{1 / 2} \boldsymbol{\nabla} \boldsymbol{u}$ and $\boldsymbol{\nabla} \boldsymbol{u}$ respectively. Indeed, the two sequences are weakly convergent to these limits, so we have:

$$
\begin{gathered}
\liminf _{n \rightarrow+\infty} \int_{\Omega \times(0, T-\epsilon)} \mu_{t}\left(k^{(n)}\right)\left|\nabla_{h} \boldsymbol{u}^{(n)}\right|^{2} \mathrm{~d} \boldsymbol{x} \mathrm{d} t \geq \int_{\Omega \times(0, T-\epsilon)} \mu_{t}(k)|\nabla \boldsymbol{u}|^{2} \mathrm{~d} \boldsymbol{x} \mathrm{d} t, \\
\liminf _{n \rightarrow+\infty} \int_{\Omega \times(0, T-\epsilon)}\left|\nabla_{h} \boldsymbol{u}^{(n)}\right|^{2} \mathrm{~d} \boldsymbol{x} \mathrm{d} t \geq \int_{\Omega \times(0, T-\epsilon)}|\boldsymbol{\nabla} \boldsymbol{u}|^{2} \mathrm{~d} \boldsymbol{x} \mathrm{d} t,
\end{gathered}
$$


but also:

$$
\begin{aligned}
& \liminf _{n \rightarrow+\infty} \int_{\Omega \times(0, T-\epsilon)}\left(\mu_{0}+\mu_{t}\left(k^{(n)}\right)\right)\left|\nabla_{h} \boldsymbol{u}^{(n)}\right|^{2} \mathrm{~d} \boldsymbol{x} \mathrm{d} t= \\
& \int_{\Omega \times(0, T-\epsilon)}\left(\mu_{0}+\mu_{t}(k)\right)|\nabla \boldsymbol{u}|^{2} \mathrm{~d} \boldsymbol{x} \mathrm{d} t,
\end{aligned}
$$

which yields the convergence of the norms, and thus the strong convergence (working on $\Omega \times(0, T-\epsilon), \forall \epsilon>0$, being sufficient to pass to the limit in the discrete turbulent energy balance, since we only consider in (22) test functions with compact support in $\Omega \times[0, T))$.

\section{REFERENCES}

[1] P. Bénilan, L. Boccardo, T. Gallouët, R. Gariepy, M. Pierre, and J.-L. Vazquez. An $L^{1}$-theory of existence and uniqueness of solutions of nonlinear elliptic equations. Annali della Scuola Normale Superiora di Pisa, Classe de Scienze, 22:240-273, 1995. (MR1354907).

[2] L. Boccardo and T. Gallouët. Non-linear elliptic and parabolic equations involving measure data. Journal of Functional Analysis, 87:149-169, 1989. (MR1025884).

[3] P. G. Ciarlet. Handbook of numerical analysis volume II : Finite elements methods - Basic error estimates for elliptic problems. In P. Ciarlet and J.L. Lions, editors, Handbook of Numerical Analysis, Volume II, pages 17-351. North Holland, 1991. (MR1115237).

[4] G. Cimatti. Existence of weak solutions for the nonstationary problem of the Joule heating of a conductor. Annali di Matematica Pura ed Applicata, 162:33-42, 1992. (MR1199645).

[5] S. Clain. Analyse mathématique et numérique d'un modèle de chauffage par induction. $\mathrm{PhD}$ thesis, EPFL, 1994.

[6] M. Crouzeix and P.-A. Raviart. Conforming and nonconforming finite element methods for solving the stationary Stokes equations I. Revue Française d'Automatique, Informatique et Recherche Opérationnelle (R.A.I.R.O.), R-3:33-75, 1973. (MR0343661).

[7] R. Eymard, T Gallouët, and R. Herbin. Finite volume methods. In P. Ciarlet and J.L. Lions, editors, Handbook of Numerical Analysis, Volume VII, pages 713-1020. North Holland, 2000. (MR1804748).

[8] R. Eymard, T Gallouët, and R. Herbin. Discretisation of heterogeneous and anisotropic diffusion problems on general nonconforming meshes - SUSHI: a scheme using stabilization and hybrid interfaces. IMA Journal of Numerical Analysis, 30:1009-1043, 2009. (MR2727814).

[9] T. Gallouët, R. Herbin, and J.-C. Latché. A convergent finite element-finite volume scheme for the compressible Stokes problem. I. The isothermal case. Math. Comp., 78(267):1333-1352, 2009.

[10] T. Gallouët, A. Larcher, and J.-C. Latché. Convergence of a finite volume scheme for the convection-diffusion equation with $L^{1}$ data. To appear in Mathematics of Computation, 2011.

[11] A. Larcher and J.-C. Latché. Convergence analysis of a finite element - finite volume scheme for a RANS turbulence model. Submitted, 2010.

[12] R. Lewandowski. The mathematical analysis of the coupling of a turbulent kinetic energy equation to the Navier-Stokes equation with an eddy viscosity. Nonlinear Analysis, Theory, Methods \& Applications, 28:393-417, 1997. (MR1418142).

[13] R. Rannacher and S. Turek. Simple nonconforming quadrilateral Stokes element. Numerical Methods for Partial Differential Equations, 8:97-111, 1992. (MR1148797).

[14] J. Simon. Compact sets in the space $L^{p}(0, T ; B)$. Annali di Matematica Pura ed Applicata, 146:65-96, 1987. (MR0916688).

[15] R. Temam. Navier-Stokes equations. volume 2 of Studies in mathematics and its applications. North Holland, 1977. (MR0609732).

E-mail address: gallouet@cmi.univ-mrs.fr

E-mail address: jean-claude.latche@irsn.fr 